\title{
Systematic review with network meta-analysis: Comparative efficacy of oral nucleos(t)ide analogues for the prevention of chemotherapy-induced hepatitis B virus reactivation
}

\author{
Min-Yue Zhang ${ }^{1, *}$, Gui-Qi Zhu ${ }^{2,3, *}$, Ke-Qing Shi', ${ }^{2,4}$ Ji-Na Zheng ${ }^{2,3}$, Zhang Cheng ${ }^{2,3}$, \\ Zhuo-Lin Zou ${ }^{5}$, Hong-Hui Huang ${ }^{1}$, Fang-Yuan Chen ${ }^{1}$, Ming-Hua Zheng ${ }^{2,4}$ \\ ${ }^{1}$ Department of Hematology, Renji Hospital, School of Medicine, Shanghai Jiaotong University, Shanghai 200127, China \\ ${ }^{2}$ Department of Hepatology, Liver Research Center, the First Affiliated Hospital of Wenzhou Medical University, Wenzhou \\ 325000, China \\ ${ }^{3}$ School of the First Clinical Medical Sciences, Wenzhou Medical University, Wenzhou 325000, China \\ ${ }^{4}$ Institute of Hepatology, Wenzhou Medical University, Wenzhou 325000, China \\ ${ }^{5}$ Department of Infection Diseases, the First Hospital of Jiaxing, Jiaxing 314000, China \\ ${ }^{*}$ Co-first authors, these authors contributed equally to this work \\ Correspondence to: Ming-Hua Zheng, e-mail: zhengmh@wmu.edu.cn \\ Fang-Yuan Chen, e-mail: chenfangyuan62@163.com \\ Keywords: hepatitis B virus reactivation, chemotherapy, nucleos(t)ide analogues prophylaxis, network meta-analysis, \\ indirect comparison \\ Received: February 28, $2016 \quad$ Accepted: April 02, $2016 \quad$ Published: April 21, 2016
}

\section{ABSTRACT}

Objectives: Currently, no consensus exists regarding the optimal oral prophylactic regimens for hepatitis $B$ surface antigen seropositive patients undergoing chemotherapy. We aimed to compare the efficacy of oral nucleos( $t$ )ide analogues (NAs), including lamivudine, entecavir, adefovir, telbivudine and tenofovir, for the prevention of chemotherapy-induced hepatitis B virus (HBV) reactivation and its related morbidity and mortality in patients with chronic HBV (CHB) infection.

Results: Fifty-two eligible articles consisting of $\mathbf{3 8 9 2}$ participants were included. For HBV reactivation, prophylactic treatment with NAs were all significantly superior to no prophylaxis, with odds ratio (OR) from 0.00 ( $95 \%$ confidence interval [CI] $0.00 \sim 0.04$ ) for the most effective intervention (tenofovir) to 0.10 ( $95 \% \mathrm{CI} 0.06 \sim 0.14$ ) for the least effective intervention (lamivudine). For secondary outcomes, prophylaxis with NAs also significantly outperformed observation. The results suggested that entecavir reduced the risk of HBV related hepatitis (predicted probability, $83 \%$ ), HBV related death $(68 \%)$ and all causes of hepatitis (97\%) most efficaciously. It ranked second in decreasing all causes of death (34\%).

Materials and Methods: PubMed, Embase and Cochrane Library database were searched for controlled trials up to March 31, 2015. Primary outcome was the incidence of $\mathrm{HBV}$ reactivation. Secondary outcomes included the incidence of HBVrelated hepatitis and death, all causes of hepatitis and death. Network meta-analysis combined direct and indirect evidence to estimate ORs for the clinical outcomes. A mean ranking and the probability of optimal therapeutic regime was obtained for each treatment based on clinical outcomes.

Conclusions: Available evidence suggests that prophylatic therapy with tenofovir and entecavir may be the most potent interventions in prevention of $\mathrm{HBV}$ reactivation and HBV-related morbidity and mortality for $\mathrm{CHB}$ infection patients undergoing chemotherapy. 


\section{INTRODUCTION}

Hepatitis B virus (HBV) infection is a public and medical issue all over the world. Approximately 240 million people show serological evidence of chronic infection (hepatitis surface antigen [HBsAg] positive) [1]. Patients with chronic HBV (CHB) infection receiving immunosuppression treatment, such as oral corticosteroids, chemotherapy, immunosuppressors or hematopoietic stem cell transplantation (HSCT), may experience the risk of $\mathrm{HBV}$ reactivation, severe hepatitis and even life-threatening hepatic failure due to the suppression of immune system and enhancement of virus replication. Previous studies reported that the incidences of chemotherapy-induced $\mathrm{HBV}$ reactivation and $\mathrm{HBV}$-related death in $\mathrm{HBsAg}$ positive patients were $19.32-85 \%$ and $2.27-33.33 \%$ [2-5], respectively. The serious complications of $\mathrm{HBV}$ reactivation in cancer patients can also cause disruption of chemotherapy, which may have negative effect on patients' survival [6].

Because of the high rate of $\mathrm{HBV}$ reactivation-related morbidity and mortality in such individuals, there has been an increase in the awareness of the importance of prophylactic anti-HBV treatment during chemotherapy. Lamivudine, an oral nucleos(t)ide analog (NA), has been widely used for treating CHB infection in the last ten years. Lamivudine may inhibit HBV replication, decrease viral load in serum and improve hepatitis both clinically and histologically. A growing number of randomized controlled trials (RCTs) or retrospective studies have demonstrated that lamivudine prophylaxis can also improve the clinical outcome of HBsAg positive cancer patients undergoing chemotherapy with few adverse effects [2, 6-8]. According to APASL 2012 guidelines for management of CHB infection, lamivudine is the first-line pophylactic anti-HBV agent recommended for HBsAg positive patients at the initiation of cancer chemotherapy and its use is recommended to continue for at least 6 months after the completion of chemotherapy [9].

Currently, available NAs for clinical intervention include entecavir, adefovir, telbivudine and tenofovir in addition to lamivudine. All of these drugs can clear HBV or inhibit viral replication and improve clinical survival. Recently, several clinical studies have compared these different anti-HBV drugs for the prevention of HBV reactivation and its complications in $\mathrm{CHB}$ patients receiving chemotherapy $[3,10,11]$. Most trials used pairwise comparisons and only evaluated the prophylactic effect of one agent against lamivudine. Opinions differ concerning which oral NA is the most efficacious for prevention of $\mathrm{HBV}$ reactivation in $\mathrm{CHB}$ infection patients receiving chemotherapy.

Theoretically, RCTs with a large number of patient samples and multiple comparator arms should be conducted to answer this issue. However, this appears to be infeasible. To our knowledge, there has been no study systematically evaluating and comparing the prophylactic effects of these five anti-viral agents up to now because of a lack of evidence from head-to-head clinical trials. Network meta-analysis, which is also known as mixed-treatment comparison, may be a latent approach to solve the above problem. Compared with traditional meta-analysis, data from both direct and indirect comparisons can be synthesized by using network meta-analysis, which allows us to jointly compare several different therapeutic regimens. In view of the limitations of previous studies, we aimed to perform a systematic review and network meta-analysis to simultaneously compare the preventive effect of five oral NAs (entecavir, adefovir, telbivudine, tenofovir and lamivudine) for the prophylactic treatment of $\mathrm{HBV}$ reactivation and $\mathrm{HBV}$ - related morbidity and mortality in $\mathrm{HBsAg}$ positive patients undergoing chemotherapy.

\section{RESULTS}

\section{Study characteristics}

Through the literature search and selection based on the criteria above, 479 articles were identified by reviewing 3245 potentially relevant publications. Then 427 publications were excluded after further assessment of the full text or abstracts. Finally 52 [2-8,10-54] articles (53 trials) with a total of 3892 patients were deemed as suitable for the meta-analysis (Figure 1). All studies were two arm trials except for three trials with multiple comparator arms. Of these included trials, there were 6 RCTs; 35 retrospective cohort trials, 12 prospective cohort trials. The patients had various cancers: both solid tumors and hematological malignancies. The patients in six studies received allogeneic HSCT (allo-HSCT). As far as study sample size was concerned, the population size involved in the studies ranged from 11 to 258 . A total of $2267 \mathrm{HBsAg}$ positive patients were assigned to receive one of the five oral NAs as prophylaxis during chemotherapy or HSCT and $1625 \mathrm{HBsAg}$ positive patients didn't receive prophylactic treatment. The prophylaxis initiated $0-7$ day prior to chemotherapy and withdrawn 1-12 months after completion of chemotherapy. Detailed characteristics of the eligible studies were outlined in Table 1. The quality assessment and scores of prospective or retrospective cohort studies were summarized in Supplementary Table 1, which indicated that the quality of included studies was reliable. The results of quality assessment of RCTs suggested low to moderate risk of bias, which were summarized in Supplementary Figure 1. The geometric distribution of controlled trials on the primary and secondary outcomes were illustrated in Figure 2. Overall low heterogeneity and no significant publication bias were found among those pairwise comparisons of different prophylactic regimens (see Table 2 and Supplementary Figure 2).

\section{HBV reactivation}

Fifty-six comparisons assessed the efficacy of the whole 6 interventions to reduce the incidence of HBV reactivation. Overall, 1174 patients $(42.43 \%)$ were assigned 
to lamivudine prophylaxis, $276(9.97 \%)$ to entecavir prophylaxis, $59(2.13 \%)$ to adefovir prophylaxis, 138 $(4.99 \%)$ to telbivudine prophylaxis, $34(1.23 \%)$ to tenofovir prophylaxis and $1086(39.25 \%)$ patients did not receive any prophylactic intervention.

Figure 3A illustrated the odds ratios (ORs) with 95\% confidence interval (CI) of outcomes obtained from the network meta-analysis. All active interventions demonstrated significant superiority over no prophylactic therapy for reducing the incidence of $\mathrm{HBV}$ reactivation. In the comparisons between different active interventions, both entecavir (OR 0.23, 95\% CI $0.10 \sim 0.49$ ) and tenofovir (OR $0.04,95 \%$ CI $0.00 \sim 0.43$ ) were significantly better than lamivudine. Tenofovir significantly provided a more favorable outcome than adefovir (OR 0.05, 95\% CI 0.00 0.71). Although statistical significance was not reached for other comparisons, there was a trend that entecavir was superior to adefovir (OR 0.29, 95\% CI $0.09 \sim 1.06$ ) and telbivudine (OR 0.53, 95\% CI $0.12 \sim 2.17$ ), while tenofovir was superior to entecavir (OR $0.17,95 \%$ CI $0.00 \sim 2.03$ ) and telbivudine (OR 0.09, 95\% CI $0.00 \sim 1.35$ ). Figure 4A-4F showed the distribution of probabilities of each prophylactic interventions being ranked at each of the possible six positions. Tenofovir had the highest probabilities $(90.0 \%)$ for $\mathrm{HBV}$ reactivation rate reduction followed by entecavir with the second highest probability $(73.0 \%)$.

\section{HBV-related hepatitis}

A total of 36 comparisons, including 5 interventions were available to this analysis. Overall, 1164 patients (42.91\%) were assigned to lamivudine prophylaxis, $178(6.56 \%)$ to entecavir prophylaxis, $35(1.29 \%)$ to adefovir prophylaxis, 124 (4.57\%) to telbivudine prophylaxis, and 1212 (44.67\%) patients did not receive any prophylactic intervention.

Figure $3 \mathrm{~B}$ showed the result of the direct and indirect comparisons of the included regimens for this outcome. Significant efficacy for all active interventions could be observed when compared with control, with the exception of adefovir. In the comparisons between four anti-HBV interventions, entecavir achieved a significantly positive clinical outcome when compared with lamivudine (OR 0.19, 95\% CI $0.05 \sim 0.56$ ). In addition, although not differing significantly, entecavir also tended to show greater beneficial effects than adefovir (OR 0.08, 95\% CI $0.00 \sim 1.36$ ) or telbivudine (OR $0.41,95 \%$ CI $0.07 \sim 2.07$ ). Figure $4 \mathrm{~A}-4 \mathrm{E}$ showed the distribution of probabilities of each prophylactic interventions being ranked at each of the possible five positions. Entecavir had the highest probabilities $(83 \%)$ of with respect to reducing the incidence of HBV-related hepatitis.

\section{HBV-related death}

Thirty-two comparisons, including 4 interventions contributed to this analysis. A total of 729 patients $(43.19 \%)$ were assigned to lamivudine prophylaxis, 85 $(5.04 \%)$ to entecavir prophylaxis, $7(0.41 \%)$ to adefovir prophylaxis and $867(51.36 \%)$ patients did not receive any prophylactic treatment.

Figure $3 \mathrm{C}$ showed the pooled estimates for the outcome of reducing the incidence of HBV-related death

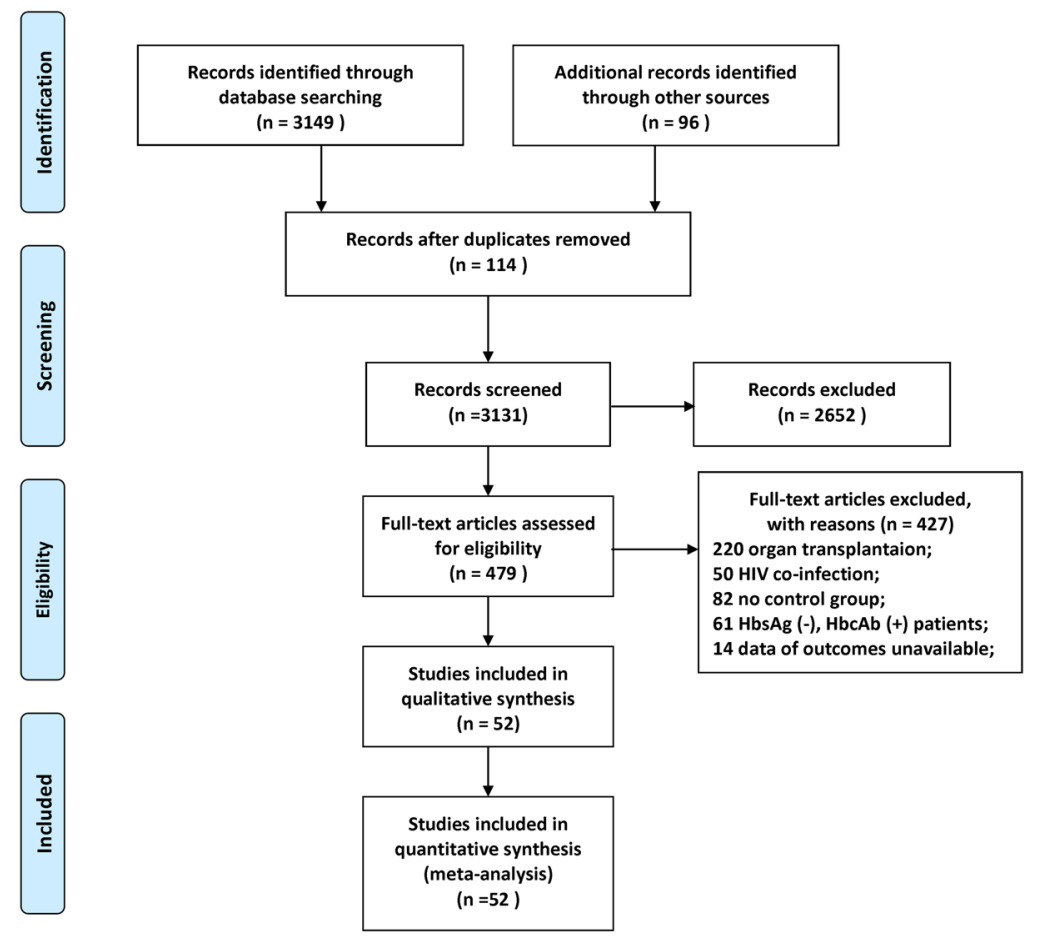

Figure 1: Literature search and selection. 


\begin{tabular}{|c|c|c|c|c|c|c|c|c|}
\hline \multirow[b]{2}{*}{$\begin{array}{l}\text { Study, Year } \\
\text { [Reference] }\end{array}$} & \multirow[b]{2}{*}{ Country } & \multirow[b]{2}{*}{$\begin{array}{l}\text { Types of } \\
\text { cancer }\end{array}$} & \multirow[b]{2}{*}{$\begin{array}{c}\text { intervention } \\
\text { A vs. B }\end{array}$} & \multirow[b]{2}{*}{$\begin{array}{c}\text { Treatment } \\
\text { duration } \\
\text { Median (range) } \\
\text { or Mean } \pm \text { SD }\end{array}$} & \multicolumn{4}{|c|}{ Intervention A vs. B } \\
\hline & & & & & $\begin{array}{l}\text { Age (y) } \\
\text { Median } \\
\text { (range) or } \\
\text { Mean } \pm \\
\text { SD }\end{array}$ & $\begin{array}{l}\text { Total } \\
\text { No. } \\
(n)\end{array}$ & $\begin{array}{l}\text { Male } \\
\text { No. } \\
(n)\end{array}$ & $\begin{array}{c}\text { Follow } \\
\text { up (m) } \\
\text { Median } \\
\text { (range) } \\
\text { or Mean } \\
\pm \text { SD }\end{array}$ \\
\hline \multicolumn{9}{|c|}{ Two-arms trials } \\
\hline \multicolumn{9}{|c|}{ Randomized controlled trials } \\
\hline $\begin{array}{l}\text { Lau et al., } \\
2003 \text { [6] }\end{array}$ & $\begin{array}{l}\text { China } \\
\text { HongKong }\end{array}$ & Lymphoma & $\begin{array}{l}\text { LAM } 100 \mathrm{mg} / \mathrm{d} \\
\text { vs. CON }\end{array}$ & $\begin{array}{l}\text { Start: } 7 \text { days prior } \\
\text { to chemo } \\
\text { End: } 6 \text { weeks after } \\
\text { the completion of } \\
\text { chemo }\end{array}$ & $\begin{array}{c}50.6 \\
(23 \sim 98) \\
\text { vs. 51.2 } \\
(24 \sim 98)\end{array}$ & $\begin{array}{c}15 \text { vs. } \\
15\end{array}$ & 8 vs. 9 & $>3$ \\
\hline $\begin{array}{l}\text { Jang et al., } \\
2006[8]\end{array}$ & Korea & $\begin{array}{l}\text { Hepatocellular } \\
\text { carcinoma }\end{array}$ & $\begin{array}{l}\text { LAM } 100 \mathrm{mg} / \mathrm{d} \\
\text { vs. CON }\end{array}$ & $\begin{array}{l}\text { Start: initiation of } \\
\text { chemo } \\
\text { End: } 12 \text { months } \\
\text { after the completion } \\
\text { of chemo }\end{array}$ & $\begin{array}{l}52.5 \pm 8.4 \\
\text { vs. } 53.2 \pm \\
\quad 9.0\end{array}$ & $\begin{array}{c}36 \text { vs. } \\
37\end{array}$ & $\begin{array}{c}30 \text { vs. } \\
31\end{array}$ & $>12$ \\
\hline $\begin{array}{l}\text { Hsu et al., } \\
2008 \text { [12] }\end{array}$ & $\begin{array}{l}\text { China } \\
\text { Taiwan }\end{array}$ & $\begin{array}{l}\text { Non-Hodgkin's } \\
\text { lymphoma }\end{array}$ & $\begin{array}{l}\text { LAM } 100 \mathrm{mg} / \mathrm{d} \\
\text { vs. CON }\end{array}$ & $\begin{array}{l}\text { Start: day } 1 \text { of } \\
\text { chemo } \\
\text { End: } 2 \text { months after } \\
\text { the completion of } \\
\text { chemo }\end{array}$ & $\begin{array}{c}50.5 \\
(32-67) \text { vs. } \\
41(20-74)\end{array}$ & $\begin{array}{c}26 \text { vs. } \\
25\end{array}$ & $\begin{array}{c}12 \text { vs. } \\
13\end{array}$ & $\begin{array}{c}33.2 \text { vs. } \\
38.6 \\
\text { (median) }\end{array}$ \\
\hline $\begin{array}{l}\text { Long et al., } \\
2011 \text { [13] }\end{array}$ & China & Breast cancer & $\begin{array}{l}\text { LAM } 100 \mathrm{mg} / \mathrm{d} \\
\text { vs. CON }\end{array}$ & $\begin{array}{l}\text { Start: } 7 \text { days prior } \\
\text { to chemo } \\
\text { End: } 12 \text { months } \\
\text { after the completion } \\
\text { of chemo }\end{array}$ & $\begin{array}{c}43(20-62) \\
\text { vs. } \\
45(29-64)\end{array}$ & $\begin{array}{c}21 \text { vs. } \\
21\end{array}$ & 0 vs. 0 & N/A \\
\hline $\begin{array}{l}\text { Huang et al., } \\
2014 \text { [10] }\end{array}$ & China & $\begin{array}{l}\text { Diffuse large } \\
\text { B-cell } \\
\text { lymphoma }\end{array}$ & $\begin{array}{l}\text { LAM } 100 \mathrm{mg} / \mathrm{d} \\
\text { vs. ETV } 0.5 \\
\mathrm{mg} / \mathrm{d}\end{array}$ & $\begin{array}{l}\text { Start: } 7 \text { days prior } \\
\text { to chemo } \\
\text { End: } 6 \text { months after } \\
\text { the completion of } \\
\text { chemo }\end{array}$ & $\begin{array}{c}44.5 \\
(25-76) \text { vs. } \\
41(19-66)\end{array}$ & $\begin{array}{c}60 \mathrm{vs} . \\
61\end{array}$ & $\begin{array}{c}37 \text { vs. } \\
31\end{array}$ & $\begin{array}{c}40.7(8.6- \\
62.3)\end{array}$ \\
\hline $\begin{array}{l}\text { Ho et al., } \\
2015 \text { [14] }\end{array}$ & $\begin{array}{l}\text { China } \\
\text { HongKong }\end{array}$ & $\begin{array}{l}\text { Hematological/ } \\
\text { solid } \\
\text { malignancies }\end{array}$ & $\begin{array}{l}\text { ADV } 10 \mathrm{mg} / \mathrm{d} \\
\text { vs. LAM } 100 \\
\mathrm{mg} / \mathrm{d}\end{array}$ & $\begin{array}{l}\text { Start: } 7 \text { days prior } \\
\text { to chemo } \\
\text { End: } 6 \text { months after } \\
\text { the completion of } \\
\text { chemo }\end{array}$ & $\begin{array}{l}51(29-76) \\
\text { vs. } 57 \\
(21-82)\end{array}$ & $\begin{array}{c}35 \text { vs. } \\
35\end{array}$ & $\begin{array}{c}21 \text { vs. } \\
22\end{array}$ & $>6$ \\
\hline \multicolumn{9}{|c|}{ Retrospective cohort study } \\
\hline $\begin{array}{l}\text { Lim et al., } \\
2002 \text { [15] }\end{array}$ & Singapore & $\begin{array}{l}\text { Hematological/ } \\
\text { solid } \\
\text { malignancies }\end{array}$ & $\begin{array}{c}\mathrm{LAM} 100 \mathrm{mg} / \mathrm{d}- \\
300 \mathrm{mg} / \mathrm{d} \mathrm{vs} . \\
\mathrm{CON}\end{array}$ & $\begin{array}{l}\text { Start: } 5 \text { days prior } \\
\text { to chemo } \\
\text { End: completion of } \\
\text { chemo }\end{array}$ & $\begin{array}{c}47.5 \\
(25-75) \mathrm{vs} . \\
54(28-75)\end{array}$ & $\begin{array}{c}16 \text { vs. } \\
19\end{array}$ & $\begin{array}{c}12 \text { vs. } \\
10\end{array}$ & $\begin{array}{c}11.5 \\
(1-41) \\
\text { vs. } 12 \\
(0.5-49)\end{array}$ \\
\hline $\begin{array}{l}\text { Persico et al., } \\
2002[16]\end{array}$ & Italy & $\begin{array}{l}\text { Non-Hodgkin's } \\
\text { lymphoma }\end{array}$ & $\begin{array}{l}\text { LAM } 100 \mathrm{mg} / \mathrm{d} \\
\text { vs. CON }\end{array}$ & $\begin{array}{l}\text { Start: during chemo } \\
\text { End: } 2 \text { months after } \\
\text { the completion of } \\
\text { chemo }\end{array}$ & $\begin{array}{l}\text { total } 45 \\
(38-61)\end{array}$ & $\begin{array}{c}3 \text { vs. } \\
18\end{array}$ & total 11 & 18 \\
\hline $\begin{array}{l}\text { Lee et al., } \\
2003 \text { [4] }\end{array}$ & Korea & $\begin{array}{l}\text { Non-Hodgkin's } \\
\text { lymphoma }\end{array}$ & $\begin{array}{l}\text { LAM } 100 \mathrm{mg} / \mathrm{d} \\
\text { vs. CON }\end{array}$ & N/A & $\begin{array}{c}44(29-68) \\
\text { vs. } \\
47.5 \\
(18-70)\end{array}$ & $\begin{array}{c}11 \mathrm{vs} . \\
20\end{array}$ & 6 vs. 13 & NA \\
\hline
\end{tabular}




\begin{tabular}{|c|c|c|c|c|c|c|c|c|}
\hline $\begin{array}{l}\text { Leaw et al., } \\
2004 \text { [17] }\end{array}$ & $\begin{array}{l}\text { China } \\
\text { Taiwan }\end{array}$ & $\begin{array}{l}\text { aggressive } \\
\text { lymphoma }\end{array}$ & $\begin{array}{l}\text { LAM } 100 \mathrm{mg} / \mathrm{d} \\
\text { vs. CON }\end{array}$ & $\begin{array}{l}\text { Start: initiation of } \\
\text { chemo } \\
\text { End: } 1 \text { months after } \\
\text { the completion of } \\
\text { chemo }\end{array}$ & N/A & $\begin{array}{c}11 \mathrm{vs} . \\
53\end{array}$ & N/A & $24(2-120)$ \\
\hline $\begin{array}{l}\text { Nagamatsu et } \\
\text { al., } 2004[5]\end{array}$ & Japan & $\begin{array}{l}\text { Hepatocellular } \\
\text { carcinoma }\end{array}$ & $\begin{array}{l}\text { LAM } 100 \mathrm{mg} / \mathrm{d} \\
\text { vs. CON }\end{array}$ & $\begin{array}{l}\text { Start: } 28 \text { days } \\
\text { prior to chemo } \\
\text { and continued } \\
\text { throughout chemo }\end{array}$ & $\begin{array}{c}44(29-68) \\
\text { vs. } 46 \\
(41-69)\end{array}$ & $\begin{array}{c}8 \text { vs. } \\
9\end{array}$ & 6 vs. 7 & N/A \\
\hline $\begin{array}{l}\text { Jia et al., } \\
2004[18]\end{array}$ & China & $\begin{array}{l}\text { Hematological/ } \\
\text { solid } \\
\text { malignancies }\end{array}$ & $\begin{array}{l}\text { LAM } 100 \mathrm{mg} / \mathrm{d} \\
\text { vs. CON }\end{array}$ & Start: before chemo & N/A & $\begin{array}{c}8 \text { vs. } \\
8\end{array}$ & Total 9 & N/A \\
\hline $\begin{array}{l}\text { Ozguroglu et } \\
\text { al., } \\
2004[19]\end{array}$ & Turkey & $\begin{array}{l}\text { Non-Hodgkin's } \\
\text { lymphoma }\end{array}$ & $\begin{array}{l}\text { LAM } 100 \mathrm{mg} / \mathrm{d} \\
\text { vs. CON }\end{array}$ & Start: before chemo & $\begin{array}{c}44(35-49) \\
\text { vs. } 42.5 \\
(14-72)\end{array}$ & $\begin{array}{c}4 \mathrm{vs} . \\
8\end{array}$ & 3 vs. 3 & N/A \\
\hline $\begin{array}{l}\text { Lim et al., } \\
2007 \text { [20] }\end{array}$ & Singapore & $\begin{array}{l}\text { Non-Hodgkin's } \\
\text { lymphoma }\end{array}$ & $\begin{array}{l}\text { LAM } 100 \mathrm{mg} / \mathrm{d} \\
\text { vs. CON }\end{array}$ & $\begin{array}{l}\text { End: } 3-6 \text { months } \\
\text { after the completion } \\
\text { of chemo }\end{array}$ & N/A & $\begin{array}{c}24 \mathrm{vs} . \\
21\end{array}$ & N/A & N/A \\
\hline $\begin{array}{l}\text { Chen et al., } \\
2008 \text { [21] }\end{array}$ & China & $\begin{array}{l}\text { Allo-HSCT } \\
\text { patients }\end{array}$ & LAM vs. CON & N/A & N/A & $\begin{array}{c}13 \text { vs. } \\
11\end{array}$ & N/A & $\begin{array}{c}28.2 \\
\text { (mean) }\end{array}$ \\
\hline $\begin{array}{l}\text { Tsutsumi et } \\
\text { al., } 2009 \text { [22] }\end{array}$ & Japan & $\begin{array}{l}\text { Non-Hodgkin's } \\
\text { lymphoma }\end{array}$ & LAM vs. CON & N/A & N/A & $\begin{array}{c}10 \mathrm{vs} . \\
15\end{array}$ & N/A & N/A \\
\hline $\begin{array}{l}\text { Eren et al., } \\
2009 \text { [23] }\end{array}$ & Turkey & $\begin{array}{l}\text { Hematological/ } \\
\text { solid } \\
\text { malignancies }\end{array}$ & $\begin{array}{l}\text { LAM } 100 \mathrm{mg} / \mathrm{d} \\
\text { vs. CON }\end{array}$ & $\begin{array}{l}\text { Start: not later than } \\
\text { the first day of } \\
\text { chemo } \\
\text { End: } 6 \text { months after } \\
\text { the completion of } \\
\text { chemo }\end{array}$ & N/A & $\begin{array}{c}40 \mathrm{vs} . \\
19\end{array}$ & $\begin{array}{c}\text { Total } \\
35\end{array}$ & N/A \\
\hline $\begin{array}{l}\text { Yeo et al., } \\
2009 \text { [24] }\end{array}$ & $\begin{array}{l}\text { China } \\
\text { Hongkong }\end{array}$ & $\begin{array}{l}\text { Diffuse large } \\
\text { B-cell } \\
\text { lymphoma }\end{array}$ & LAM vs. CON & N/A & N/A & $\begin{array}{c}15 \\
\text { Vs. } 9\end{array}$ & N/A & N/A \\
\hline $\begin{array}{l}\text { Koo et al., } \\
2010[25]\end{array}$ & Singapore & $\begin{array}{l}\text { Non-Hodgkin's } \\
\text { lymphoma }\end{array}$ & LAM vs. CON & N/A & N/A & $\begin{array}{c}18 \\
\text { vs. } 8\end{array}$ & N/A & N/A \\
\hline $\begin{array}{l}\text { Topcuoglu } \\
\text { et al., } \\
2010[26]\end{array}$ & Turkey & $\begin{array}{l}\text { Allo-HSCT } \\
\text { patients }\end{array}$ & $\begin{array}{l}\mathrm{LAM} 100 \mathrm{mg} / \mathrm{d} \\
\text { vs. CON }\end{array}$ & $\begin{array}{l}\text { Start: initiated } \\
\text { with conditioning } \\
\text { regimen } \\
\text { End: 6-12 } \\
\text { month after the } \\
\text { cessation of } \\
\text { immunosuppresion } \\
\text { at posttransplant } \\
\text { period }\end{array}$ & $\begin{array}{c}\text { Total } \\
\text { median } 33\end{array}$ & $\begin{array}{c}14 \\
\text { vs. } 9\end{array}$ & $\begin{array}{c}\text { Total } \\
22\end{array}$ & N/A \\
\hline $\begin{array}{l}\text { Pei et al., } \\
2010 \text { [27] }\end{array}$ & $\begin{array}{l}\text { China } \\
\text { Taiwan }\end{array}$ & $\begin{array}{l}\text { Non-Hodgkin's } \\
\text { lymphoma }\end{array}$ & LAM vs. CON & $\begin{array}{l}\text { Start: prior to } \\
\text { chemo } \\
\text { End: } 0-9 \text { month } \\
\text { (range) months } \\
\text { after cessation } \\
\text { of rituximab } \\
\text { treatment. (median } \\
\text { 2month) }\end{array}$ & $\begin{array}{c}49(31-72) \\
\text { vs. } \\
54(40-81)\end{array}$ & $\begin{array}{l}5 \mathrm{vs} \\
.10\end{array}$ & 2 vs. 5 & N/A \\
\hline $\begin{array}{l}\text { Sohn et al., } \\
2011 \text { [28] }\end{array}$ & Korea & Breast cancer & LAM vs. CON & $\begin{array}{l}\text { before or during } \\
\text { chemo prior to } \\
\text { development of an } \\
\text { apparent clinical } \\
\text { hepatitis flare-up }\end{array}$ & $\begin{array}{c}48(29-66) \\
\text { vs. } \\
46(23-75)\end{array}$ & $\begin{array}{c}41 \mathrm{vs} . \\
128\end{array}$ & 0 vs. 0 & N/A \\
\hline $\begin{array}{l}\text { Yun et al., } \\
2011 \text { [29] }\end{array}$ & Korea & Breast cancer & $\begin{array}{l}\text { LAM } 100 \mathrm{mg} / \mathrm{d} \\
\text { vs. CON }\end{array}$ & $\begin{array}{l}\text { Start: within } 7 \text { days } \\
\text { prior to chemo }\end{array}$ & $\begin{array}{c}48(30-68) \\
\text { vs. } \\
46(30-69)\end{array}$ & $\begin{array}{l}55 \mathrm{vs} . \\
76\end{array}$ & 0 vs. 0 & N/A \\
\hline
\end{tabular}




\begin{tabular}{|c|c|c|c|c|c|c|c|c|}
\hline $\begin{array}{l}\text { Yan et al., } \\
2012[30]\end{array}$ & China & Lung cancer & LAM vs. CON & $\begin{array}{l}\text { Start: initiation of } \\
\text { chemo }\end{array}$ & N/A & $\begin{array}{c}33 \text { vs. } \\
43\end{array}$ & $\begin{array}{c}27 \text { vs. } \\
34\end{array}$ & N/A \\
\hline $\begin{array}{l}\text { Mya et al., } \\
2012 \text { [31] }\end{array}$ & Singapore & $\begin{array}{l}\text { multiple } \\
\text { myeloma }\end{array}$ & $\begin{array}{l}\text { LAM } 100 \mathrm{mg} / \mathrm{d} \\
\text { vs. CON }\end{array}$ & $\begin{array}{l}\text { Start: before chemo } \\
\text { End: } 6-12 \text { months } \\
\text { after the completion } \\
\text { of chemo }\end{array}$ & N/A & $\begin{array}{c}11 \\
\text { vs. } 4\end{array}$ & N/A & $\begin{array}{c}\text { median } \\
33.6\end{array}$ \\
\hline $\begin{array}{l}\text { Chen et al., } \\
2012 \text { [32] }\end{array}$ & China & $\begin{array}{l}\text { Diffuse large } \\
\text { B-cell } \\
\text { lymphoma }\end{array}$ & $\begin{array}{l}\text { LAM } 100 \mathrm{mg} / \mathrm{d} \\
\text { vs. CON }\end{array}$ & $\begin{array}{l}\text { Start: } 7 \text { days prior } \\
\text { to chemo } \\
\text { End: } 3 \text { months after } \\
\text { the completion of } \\
\text { chemo }\end{array}$ & $\begin{array}{c}47(21-76) \\
\text { vs. } \\
46.9 \\
(22-76)\end{array}$ & $\begin{array}{l}30 \text { vs. } \\
20\end{array}$ & $\begin{array}{c}19 \text { vs. } \\
11\end{array}$ & N/A \\
\hline $\begin{array}{l}\text { Wang et al., } \\
2013 \text { [33] }\end{array}$ & China & $\begin{array}{l}\text { Hematological/ } \\
\text { solid } \\
\text { malignancies }\end{array}$ & LAM vs. CON & N/A & N/A & $\begin{array}{c}47 \mathrm{vs} . \\
113\end{array}$ & N/A & N/A \\
\hline $\begin{array}{l}\text { Lin et al., } \\
2014 \text { [2] }\end{array}$ & China & Lung cancer & $\begin{array}{l}\text { LAM } 100 \mathrm{mg} / \mathrm{d} \\
\text { vs. CON }\end{array}$ & $\begin{array}{l}\text { Start: } 7 \text { days prior } \\
\text { to chemo } \\
\text { End: } 3 \text { months } \\
\text { after completion of } \\
\text { chemo }\end{array}$ & $\begin{array}{c}61.5 \\
(34-77) \text { vs. } \\
59(30-79)\end{array}$ & $\begin{array}{c}82 \text { vs. } \\
176\end{array}$ & $\begin{array}{c}49 \text { vs. } \\
107\end{array}$ & N/A \\
\hline $\begin{array}{l}\text { Lee et al., } \\
2014 \text { [7] }\end{array}$ & Korea & Breast cancer & $\begin{array}{l}\text { LAM } 100 \mathrm{mg} / \mathrm{d} \\
\text { vs. CON }\end{array}$ & $\begin{array}{l}\text { End: } 0-28.9 \text { month } \\
\text { after the completion } \\
\text { of chemo; median } \\
\text { duration } 7.5 \mathrm{~m} \\
(2.1-34.7 \mathrm{~m})\end{array}$ & $\begin{array}{c}46(29-67) \\
\text { Vs. } \\
45(29-72)\end{array}$ & $\begin{array}{c}73 \\
\text { vs.92 }\end{array}$ & 0 vs. 0 & $\begin{array}{c}49.7 \\
(16.1- \\
121.3) \mathrm{vs} . \\
74(23.5- \\
140.6)\end{array}$ \\
\hline $\begin{array}{l}\text { Nishida et al., } \\
2013 \text { [34] }\end{array}$ & Japan & $\begin{array}{l}\text { Hematological/ } \\
\text { solid } \\
\text { malignancies }\end{array}$ & ETV vs. CON & N/A & $\begin{array}{c}60(43-79) \\
\text { vs. } \\
59(36-74)\end{array}$ & $\begin{array}{c}8 \mathrm{vs} . \\
29\end{array}$ & 2 vs. 10 & $\begin{array}{c}25(2-32) \\
\text { vs. } \\
19(4-102)\end{array}$ \\
\hline $\begin{array}{l}\text { Li et al., } \\
2011[36]\end{array}$ & China & Lymphoma & $\begin{array}{l}\text { LAM } 100 \mathrm{mg} / \mathrm{d} \\
\text { vs. ETV } 0.5 \\
\text { mg/d }\end{array}$ & $\begin{array}{l}\text { Start: } 7 \text { days prior } \\
\text { to chemo }\end{array}$ & $\begin{array}{c}46(20-81) \\
\text { VS. } 44 \\
(17-74) \\
\end{array}$ & $\begin{array}{c}89 \text { vs. } \\
34\end{array}$ & $\begin{array}{c}52 \text { vs. } \\
22\end{array}$ & N/A \\
\hline $\begin{array}{l}\text { Min et al., } \\
2012 \text { [37] }\end{array}$ & Korea & $\begin{array}{l}\text { non-hepatic } \\
\text { cancer }\end{array}$ & LAM vs. ETV & $\begin{array}{l}11.1 \pm 8.2 \text { vs. } 11.5 \\
\pm 6.9\end{array}$ & $\begin{array}{c}51.5 \pm 9.4 \\
\text { vs. } \\
48.2 \pm 9.4\end{array}$ & $\begin{array}{c}146 \\
\text { vs. } 40\end{array}$ & N/A & N/A \\
\hline $\begin{array}{l}\text { Chen et al., } \\
2013 \text { [35] }\end{array}$ & Australia & $\begin{array}{l}\text { Haematological } \\
\text { malignancies }\end{array}$ & $\begin{array}{l}\text { LAM } 100 \mathrm{mg} / \mathrm{d} \\
\text { vs. ETV } 0.5 \\
\mathrm{mg} / \mathrm{d}\end{array}$ & N/A & N/A & $\begin{array}{c}11 \\
\text { vs. } 4\end{array}$ & N/A & 36 \\
\hline $\begin{array}{l}\text { Ling et al., } \\
2013 \text { [38] }\end{array}$ & Singapore & $\begin{array}{l}\text { Solid } \\
\text { malignancies }\end{array}$ & LAM vs. ETV & N/A & N/A & $\begin{array}{c}24 \\
\text { vs. } 4 \\
\end{array}$ & N/A & N/A \\
\hline \multicolumn{9}{|c|}{ Retrospective cohort study with historical control group } \\
\hline $\begin{array}{l}\text { Lau et al., } \\
2003 \text { [39] }\end{array}$ & $\begin{array}{l}\text { China } \\
\text { Hongkong }\end{array}$ & $\begin{array}{l}\text { Allo-HSCT } \\
\text { patients }\end{array}$ & $\begin{array}{l}\text { LAM } 100 \mathrm{mg} / \mathrm{d} \\
\text { vs. CON }\end{array}$ & & $\begin{array}{c}38.5 \\
(13-54) \text { vs. } \\
32(5-48) \\
\end{array}$ & $\begin{array}{c}20 \text { vs. } \\
20\end{array}$ & $\begin{array}{c}10 \mathrm{vs} . \\
16\end{array}$ & $>12$ \\
\hline $\begin{array}{l}\text { Li et al., } \\
2006[40]\end{array}$ & China & Lymphoma & $\begin{array}{l}\text { LAM } 100 \mathrm{mg} / \mathrm{d} \\
\text { vs. CON }\end{array}$ & $\begin{array}{l}\text { Start: } 7 \text { days prior } \\
\text { to chemo } \\
\text { End: } 8 \text { weeks after } \\
\text { the completion of } \\
\text { chemo }\end{array}$ & $\begin{array}{c}40(16-74) \\
\text { vs. } 41 \\
(12-75)\end{array}$ & $\begin{array}{c}40 \mathrm{vs} . \\
116\end{array}$ & $\begin{array}{l}26 \text { vs. } \\
72\end{array}$ & $>3$ \\
\hline $\begin{array}{l}\text { Hsiao et al., } \\
2006 \text { [41] }\end{array}$ & $\begin{array}{l}\text { China } \\
\text { Taiwan }\end{array}$ & $\begin{array}{l}\text { Allo-HSCT } \\
\text { patients }\end{array}$ & $\begin{array}{l}\text { LAM } 100 \mathrm{mg} / \mathrm{d} \\
\text { vs. CON }\end{array}$ & $\begin{array}{l}\text { Start: } 0-62 \text { weeks } \\
\text { (range) prior to } \\
\text { allo-HSCT, } 11 \\
\text { weeks (median) } \\
\text { End: } \\
\text { posttransplantation } \\
\text { period }\end{array}$ & $\begin{array}{l}41(19-56) \\
(\text { LAM })\end{array}$ & $\begin{array}{l}16 \mathrm{vs} . \\
55\end{array}$ & $\begin{array}{c}12 \\
(\mathrm{LAM})\end{array}$ & $39(2-216)$ \\
\hline
\end{tabular}




\begin{tabular}{|c|c|c|c|c|c|c|c|c|}
\hline $\begin{array}{l}\text { Cil et al., } \\
2008 \text { [42] }\end{array}$ & Turkey & $\begin{array}{l}\text { Hematological/ } \\
\text { solid } \\
\text { malignancies }\end{array}$ & $\begin{array}{l}\text { LAM } 100 \mathrm{mg} / \mathrm{d} \\
\text { vs. CON }\end{array}$ & $\begin{array}{l}\text { Start: } 7 \text { days prior } \\
\text { to chemo } \\
\text { End: } 2 \text { months after } \\
\text { the completion of } \\
\text { chemo }\end{array}$ & $\begin{array}{c}44(22-66) \\
\text { vs. } \\
46(24-70)\end{array}$ & $\begin{array}{l}37 \mathrm{vs} . \\
50\end{array}$ & $\begin{array}{c}23 \text { vs. } \\
32\end{array}$ & 31 (LAM) \\
\hline $\begin{array}{l}\text { Huang et al., } \\
2009 \text { [43] }\end{array}$ & China & $\begin{array}{l}\text { Allo-HSCT } \\
\text { patients }\end{array}$ & $\begin{array}{l}\text { LAM } 100 \mathrm{mg} / \mathrm{d} \\
\text { vs. CON }\end{array}$ & $\begin{array}{l}\text { Start: } 7 \text { days prior } \\
\text { to chemo } \\
\text { End: } 6 \text { months after } \\
\text { the completion of } \\
\text { allo-HSCT }\end{array}$ & $\begin{array}{c}37 \pm 12 \text { vs } \\
29 \pm 9\end{array}$ & $\begin{array}{c}20 \mathrm{vs} . \\
12\end{array}$ & 13 vs 7 & $\begin{array}{c}12.3 \text { vs. } \\
43.8 \\
\text { (median) }\end{array}$ \\
\hline \multicolumn{9}{|c|}{ Prospective cohort studies } \\
\hline $\begin{array}{l}\text { Shibole et al., } \\
2002[44]\end{array}$ & Isreal & Lymphoma & $\begin{array}{l}\text { LAM } 150 \mathrm{mg} / \mathrm{d} \\
\text { vs. CON }\end{array}$ & $\begin{array}{l}\text { Start: prior to } \\
\text { initiation of chemo } \\
\text { End: } 6 \text { months after } \\
\text { the completion of } \\
\text { chemo }\end{array}$ & $\begin{array}{c}55(38-65) \\
\text { vs. } \\
57.5 \\
(46-67)\end{array}$ & $\begin{array}{c}7 \text { vs. } \\
4\end{array}$ & 4 vs. 4 & N/A \\
\hline $\begin{array}{l}\text { Idilman et al., } \\
2004 \text { [45] }\end{array}$ & Turkey & $\begin{array}{l}\text { Hematological/ } \\
\text { solid } \\
\text { malignancies }\end{array}$ & $\begin{array}{l}\text { LAM } 100 \mathrm{mg} / \mathrm{d} \\
\text { vs. CON }\end{array}$ & $\begin{array}{l}\text { Start: initiation of } \\
\text { chemo } \\
\text { End: } 12 \text { months } \\
\text { after the completion } \\
\text { of chemo }\end{array}$ & $\begin{array}{c}42(35-68) \\
\text { vs. } \\
40(25-51)\end{array}$ & $\begin{array}{c}8 \mathrm{vs} . \\
10\end{array}$ & 5 vs. 4 & $\begin{array}{c}17.1 \\
(8-29) \\
\text { vs. } 32.1 \\
(5-59)\end{array}$ \\
\hline $\begin{array}{l}\text { Tsai et al., } \\
2011 \text { [46] }\end{array}$ & $\begin{array}{l}\text { China } \\
\text { Taiwan }\end{array}$ & breast cancer & $\begin{array}{l}\text { LAM } 100 \mathrm{mg} / \mathrm{d} \\
\text { vs. CON }\end{array}$ & $\begin{array}{l}\text { Start: } 7 \text { days prior } \\
\text { to chemo } \\
\text { End: } 1 \text { months after } \\
\text { the completion of } \\
\text { chemo }\end{array}$ & $\begin{array}{l}46.7 \pm 9.2 \\
\text { vs. } 50.4 \pm \\
\quad 7.7\end{array}$ & $\begin{array}{c}23 \text { vs. } \\
22\end{array}$ & 0 vs. 0 & $>3$ \\
\hline $\begin{array}{l}\text { Kim et al., } \\
2013 \text { [3] }\end{array}$ & Asian & $\begin{array}{l}\text { B-cell } \\
\text { lymphoma }\end{array}$ & LAM vs. ETV & N/A & N/A & $\begin{array}{c}28 \text { vs. } \\
16\end{array}$ & l & N/A \\
\hline $\begin{array}{l}\text { Chen et al., } \\
2013 \text { [11] }\end{array}$ & $\begin{array}{l}\text { China } \\
\text { Taiwan }\end{array}$ & $\begin{array}{l}\text { Hematological/ } \\
\text { solid } \\
\text { malignancies }\end{array}$ & LdT vs. ETV & $\begin{array}{l}\text { Start: } 7 \text { days prior } \\
\text { to chemo }\end{array}$ & $\begin{array}{c}53.9 \pm 13.2 \\
\text { vs. } 57.3 \pm \\
13.9\end{array}$ & $\begin{array}{c}48 \text { vs. } \\
24\end{array}$ & $\begin{array}{c}22 \text { vs. } \\
15\end{array}$ & $\begin{array}{c}\text { mean } 10.7 \\
(0.57- \\
27.8)\end{array}$ \\
\hline $\begin{array}{l}\text { Gentile et al., } \\
2014 \text { [47] }\end{array}$ & Italy & $\begin{array}{l}\text { Haematological } \\
\text { malignancies }\end{array}$ & LAM vs. TDF & $\begin{array}{l}32(9-72) \text { vs. } 24 \\
(4-48)\end{array}$ & N/A & $\begin{array}{l}13 \mathrm{vs} . \\
25\end{array}$ & N/A & N/A \\
\hline \multicolumn{9}{|c|}{ Prospective cohort studies with historical control group } \\
\hline $\begin{array}{l}\text { Yeo et al., } \\
2004 \text { [48] }\end{array}$ & $\begin{array}{l}\text { China } \\
\text { Hongkong }\end{array}$ & $\begin{array}{l}\text { Hematological/ } \\
\text { solid } \\
\text { malignancies }\end{array}$ & $\begin{array}{l}\text { LAM } 100 \mathrm{mg} / \mathrm{d} \\
\text { vs. CON }\end{array}$ & $\begin{array}{l}\text { Start: } 7 \text { days prior } \\
\text { to chemo } \\
\text { End: } 2 \text { months } \\
\text { after completion of } \\
\text { chemo }\end{array}$ & $\begin{array}{c}49(35-77) \\
\text { vs. } \\
49(20-78)\end{array}$ & $\begin{array}{c}65 \mathrm{vs} . \\
193\end{array}$ & $\begin{array}{c}34 \text { vs. } \\
82\end{array}$ & $\begin{array}{l}2 \text { months } \\
\text { after the } \\
\text { completion } \\
\text { of chemo }\end{array}$ \\
\hline $\begin{array}{l}\text { Yeo et al., } \\
2004 \text { [49] }\end{array}$ & $\begin{array}{l}\text { China } \\
\text { Hongkong }\end{array}$ & Breast cancer & $\begin{array}{l}\text { LAM } 100 \mathrm{mg} / \mathrm{d} \\
\text { vs. CON }\end{array}$ & $\begin{array}{l}\text { Start: } 7 \text { days prior } \\
\text { to chemo } \\
\text { End: } 2 \text { months } \\
\text { after completion of } \\
\text { chemo }\end{array}$ & $\begin{array}{c}46(31-68) \\
\text { vs. } \\
46(31-71)\end{array}$ & $\begin{array}{l}31 \mathrm{vs} . \\
61\end{array}$ & 0 vs. 0 & $\begin{array}{l}2 \text { months } \\
\text { after the } \\
\text { completion } \\
\text { of chemo }\end{array}$ \\
\hline $\begin{array}{l}\text { Dai et al., } \\
2004[50]\end{array}$ & $\begin{array}{l}\text { China } \\
\text { Taiwan }\end{array}$ & Breast cancer & $\begin{array}{l}\text { LAM } 100 \mathrm{mg} / \mathrm{d} \\
\text { vs. CON }\end{array}$ & $\begin{array}{l}\text { Start: } 7 \text { days prior } \\
\text { to chemo } \\
\text { End: } 1 \text { months } \\
\text { after completion of } \\
\text { chemo }\end{array}$ & $\begin{array}{c}47(36-58) \\
\text { vs. } \\
43(27-55)\end{array}$ & $\begin{array}{c}11 \\
\text { vs. } 9\end{array}$ & 0 vs. 0 & $\begin{array}{c}19(11-25) \\
\text { vs. } 10 \\
(3-18)\end{array}$ \\
\hline $\begin{array}{l}\text { Yeo et al., } \\
2005 \text { [51] }\end{array}$ & $\begin{array}{l}\text { China } \\
\text { Hongkong }\end{array}$ & $\begin{array}{l}\text { Nasopharyngeal } \\
\text { Carcinoma }\end{array}$ & $\begin{array}{l}\text { LAM } 100 \mathrm{mg} / \mathrm{d} \\
\text { vs. CON }\end{array}$ & $\begin{array}{l}\text { Start: } 7 \text { days prior } \\
\text { to chemo } \\
\text { End: } 2 \text { months } \\
\text { after completion of } \\
\text { chemo }\end{array}$ & $\begin{array}{c}46.5 \\
(30-58) \text { vs. } \\
46(40-65)\end{array}$ & $\begin{array}{c}16 \text { vs. } \\
21\end{array}$ & $\begin{array}{l}14 \text { vs. } \\
15\end{array}$ & $\begin{array}{l}2 \text { months } \\
\text { after the } \\
\text { completion } \\
\text { of chemo }\end{array}$ \\
\hline
\end{tabular}




\begin{tabular}{|c|c|c|c|c|c|c|c|c|}
\hline $\begin{array}{l}\text { Hui et al., } \\
2005 \text { [52] }\end{array}$ & $\begin{array}{l}\text { China } \\
\text { Hongkong }\end{array}$ & $\begin{array}{l}\text { Allo-HSCT } \\
\text { patients }\end{array}$ & LAM vs. CON & $\begin{array}{l}\text { Start: } 7 \text { days prior } \\
\text { to allo-HSCT } \\
\text { End: } 52 \text { weeks after } \\
\text { allo-HSCT or until } \\
\text { death }\end{array}$ & $\begin{array}{l}42(23-38) \\
(\text { LAM })\end{array}$ & $\begin{array}{c}19 \text { vs. } \\
14\end{array}$ & $\begin{array}{c}10 \\
(\mathrm{LAM})\end{array}$ & N/A \\
\hline \multicolumn{9}{|c|}{ Multi-arms trials } \\
\hline \multicolumn{9}{|c|}{ Retrospective cohort study } \\
\hline $\begin{array}{l}\text { Kim et al., } \\
2013 \text { [3] }\end{array}$ & Asian & $\begin{array}{l}\text { B-cell } \\
\text { lymphoma }\end{array}$ & $\begin{array}{l}\text { ADV vs. ETV } \\
\text { vs. LAM vs. } \\
\text { CON }\end{array}$ & N/A & N/A & $\begin{array}{l}7 \text { vs. } \\
31 \text { vs. } \\
96 \text { vs. } \\
22\end{array}$ & N/A & N/A \\
\hline $\begin{array}{l}\text { Choi et al., } \\
2014 \text { [53] }\end{array}$ & Korea & $\begin{array}{l}\text { Hematological/ } \\
\text { solid } \\
\text { malignancies }\end{array}$ & $\begin{array}{l}\text { LAM vs. ETV } \\
\text { vs. ADV vs. } \\
\text { LDT vs. TDF }\end{array}$ & N/A & N/A & $\begin{array}{l}77 \text { vs. } \\
87 \\
\text { vs. } 17 \\
\text { vs. } 14 \\
\text { vs. } 9\end{array}$ & N/A & $\begin{array}{l}\text { median } \\
16.4 \\
\text { months } \\
\text { following } \\
\text { the start of } \\
\text { chemo }\end{array}$ \\
\hline \multicolumn{9}{|c|}{ Prospective cohort study } \\
\hline $\begin{array}{l}\text { Yoo et al., } \\
2012 \text { [54] }\end{array}$ & Korea & $\begin{array}{l}\text { Hematological/ } \\
\text { solid } \\
\text { malignancies }\end{array}$ & $\begin{array}{l}\text { LAM vs. ETV } \\
\text { vs. LDT }\end{array}$ & $\mathrm{N} / \mathrm{A}$ & N/A & $\begin{array}{c}86 \text { vs. } \\
31 \text { vs. } \\
124\end{array}$ & $\begin{array}{c}\text { Total } \\
129\end{array}$ & $>6$ \\
\hline
\end{tabular}

$\mathrm{CON}=$ control (no prophylaxis) LAM = lamivudine; $\mathrm{ETV}=$ entecavir; $\mathrm{ADV}=$ adefovir; $\mathrm{LdT}=$ telbivudine; $\mathrm{TDF}=$ tenofovir; Allo-HSCT $=$ allogeneic hematopoietic stem cell transplantation.

in network meta-analysis. Both lamivudine (OR 0.35, $95 \%$ CI $0.21 \sim 0.60$ ) and entecavir (OR 0.11, 95\% CI $0.01 \sim 0.58)$ showed significant superiority over control. Although not differing significantly, adefovir also appeared to be more favorable than control (OR 0.28, $95 \%$ CI $0.01 \sim 2.36)$. When compared with each active intervention, none of the comparisons reached statistical significance. However, entecavir was more likely to induce a more favorable clinical outcome than lamivudine (OR $0.32,95 \%$ CI $0.04 \sim 1.65$ ) or adefovir (OR $0.42,95 \%$ CI $0.02 \sim 18.35)$. Figure 4A-4D showed the distribution of probabilities of each prophylactic interventions being
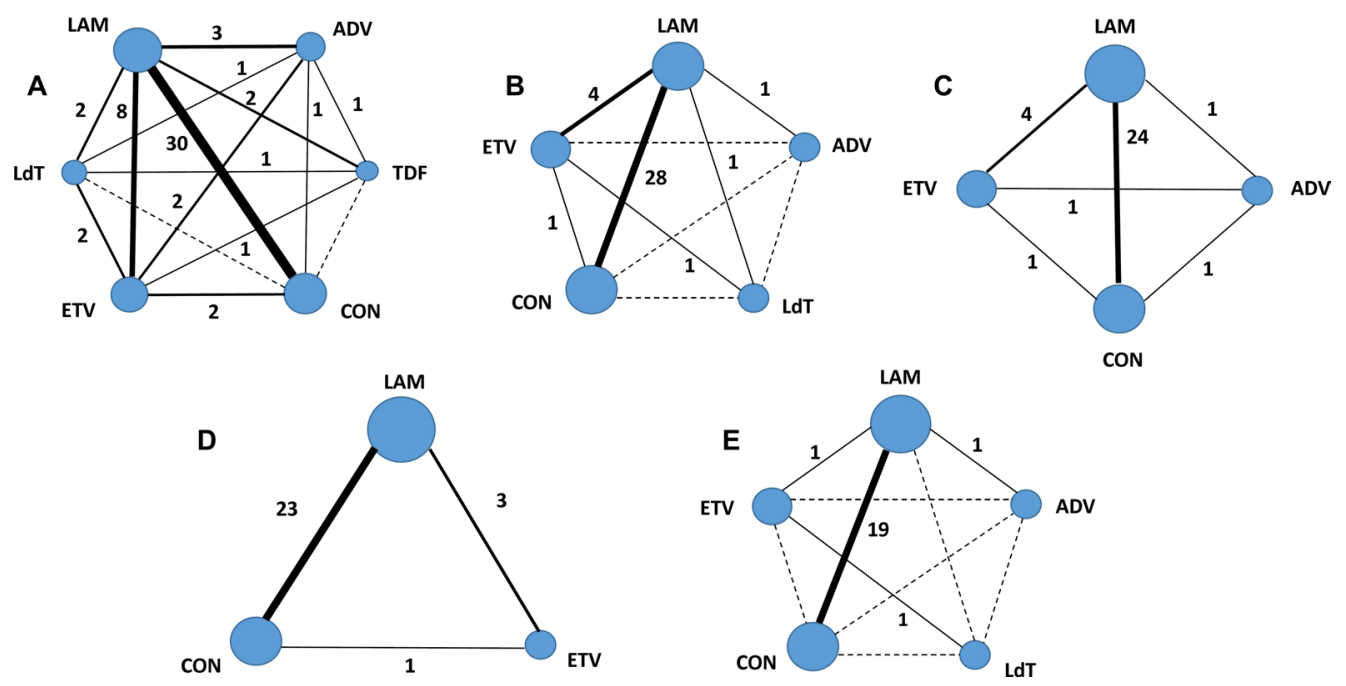

Figure 2: Evidence network of eligible comparisons for network meta-analysis. The numbers along the link lines demonstrate the number of trials or pairs of trial arms. Each solid line represents direct comparisons between regimens that have been studied in head-to head (direct) comparisons in the eligible controlled trials. Each dashed line indicates that there is no direct comparison (indirect comparison) between two regimens. The width of the lines reflects the cumulative number of trials for each comparison and the size of every cycle is proportional to the number of included regimens (sample size). Different cycles represent different regimens accordingly. (A) HBV reactivation; (B) HBV-related hepatitis; (C) HBV-related death; (D) All causes of hepatitis; (E) All causes of death. CON = control (no prophylaxis); LAM = lamivudine; $\mathrm{ETV}=$ entecavir; $\mathrm{ADV}=$ adefovir; $\mathrm{LdT}=$ telbivudine; $\mathrm{TDF}=$ tenofovir. 
Table 2: Assessment of heterogeneity for direct comparisons and comparison of outcomes between pair-wise meta-analysis and network meta-analysis

Treatment Comparisons $\quad$ Results of Pair-Wise Meta-Analysis $\quad I^{2}(\%) \quad$ Results of Network Meta-Analysis

\begin{tabular}{|c|c|c|c|}
\hline \multicolumn{4}{|c|}{ HBV reactivation } \\
\hline ETV vs. ADV & $0.38(0.02,6.10)$ & 56.3 & $0.29(0.09,1.06)$ \\
\hline LAM vs. ADV & $1.36(0.61,3.03)$ & 0 & $1.30(0.49,3.81)$ \\
\hline LAM vs. ETV & $3.67(1.72,7.82)$ & 35.2 & $4.38(2.06,9.53)$ \\
\hline ETV vs. CON & $0.19(0.00,18.73)$ & 86.8 & $0.02(0.01,0.05)$ \\
\hline LdT vs. ETV & $1.60(0.17,15.42)$ & 100 & $1.89(0.46,8.50)$ \\
\hline TDF vs. ETV & $0.98(0.05,19.57)$ & N/A & $0.17(0.00,2.03)$ \\
\hline CON vs. LAM & $7.47(5.30,10.52)$ & 0 & $10.47(7.03,16.55)$ \\
\hline LdT vs. LAM & $0.44(0.11,1.83)$ & 40.5 & $0.43(0.12,1.74)$ \\
\hline TDF vs. LAM & $0.07(0.01,0.66)$ & N/A & $0.04(0.00,0.43)$ \\
\hline \multicolumn{4}{|c|}{ HBV-related hepatitis } \\
\hline ADV vs. LAM & $2.06(0.18,23.83)$ & N/A & $2.21(0.17,82.04)$ \\
\hline LAM vs. ETV & $4.09(1.13,14.77)$ & 8.4 & $5.36(1.78,19.97)$ \\
\hline CON vs. ETV & $0.48(0.04,6.11)$ & N/A & $46.71(14.33,185.10)$ \\
\hline LdT vs. ETV & $4.60(0.26,81.82)$ & N/A & $2.43(0.48,14.64)$ \\
\hline CON vs. LAM & $6.89(4.83,9.83)$ & 0 & $8.59(5.84,13.35)$ \\
\hline LdT vs. LAM & $0.39(0.15,0.98)$ & N/A & $0.43(0.12,1.88)$ \\
\hline \multicolumn{4}{|c|}{ HBV- related death } \\
\hline LAM vs. ADV & $0.73(0.04,14.88)$ & N/A & $1.26(0.15,34.78)$ \\
\hline CON vs. ADV & $1.76(0.17,18.32)$ & N/A & $3.60(0.42,96.28)$ \\
\hline ETV vs. LAM & $1.32(0.12,15.03)$ & N/A & $0.32(0.04,1.65)$ \\
\hline CON vs. ETV & $8.22(0.95,81.99)$ & N/A & $8.88(1.73,90.38)$ \\
\hline CON vs. LAM & $2.51(1.54,4.08)$ & 0 & $2.84(1.66,4.86)$ \\
\hline \multicolumn{4}{|c|}{ All causes of hepatitis } \\
\hline LAM vs. ETV & $3.44(1.51,7.82)$ & 0 & $2.81(0.92,8.09)$ \\
\hline CON vs. ETV & $0.48(0.04,6.11)$ & N/A & $12.12(4.00,39.36)$ \\
\hline CON vs. LAM & $3.96(2.79,5.63)$ & 29.9 & $4.34(3.01,6.73)$ \\
\hline \multicolumn{4}{|c|}{ All causes of death } \\
\hline LAM vs. ADV & $1.00(0.36,2.74)$ & N/A & $1.01(0.18,5.71)$ \\
\hline LAM vs. ETV & $0.76(0.07,8.65)$ & N/A & $0.95(0.06,35.24)$ \\
\hline ETV vs. LdT & $1.68(0.57,4.97)$ & N/A & $1.72(0.28,10.02)$ \\
\hline CON vs. LAM & $2.35(1.51,3.66)$ & 11.8 & $2.83(1.68,5.01)$ \\
\hline
\end{tabular}

$\mathrm{CON}=$ control (no prophylaxis); LAM = lamivudine; ETV = entecavir; ADV = adefovir; $\mathrm{LdT}=$ telbivudine; $\mathrm{TDF}=$ tenofovir.

ranked at each of the possible four positions. Entecavir was ranked the most efficacious intervention with regard to reducing the incidence of death due to $\mathrm{HBV}$ reactivation.

\section{All causes of hepatitis}

Twenty-seven comparisons, including 3 interventions contributed to analyze the outcome of all causes of hepatitis. 
A total of 918 patients $(41.33 \%)$ were assigned to lamivudine prophylaxis, $147(6.62 \%)$ to entecavir prophylaxis and 1156 $(52.05 \%)$ patients did not receive any prophylactic regimes.

Figure 3D showed the result of the network metaanalysis for this outcome. Patients receiving lamivudine (OR $0.23,95 \%$ CI $0.15 \sim 0.33$ ) or entecavir (OR 0.08, 95\% CI $0.03 \sim 0.25)$ prophylactic treatment could significantly prevent the incidence of hepatitis when compared with no prophylactic intervention. Entecavir was associated with a superior outcome over lamivudine (OR 0.36, 95\% CI 0.12 1.08) although this did not achieve statistical significance. Figure 4A-4C showed the distribution of probabilities of each prophylactic interventions being ranked at each of the possible three positions. On the whole, entecavir was demonstrated to be the most efficacious treatment among three interventions as entecavir had the highest probabilities $(97 \%)$ in terms of hepatitis rate reduction as shown in Figure 4C.

\section{All causes of death}

A total of 22 comparisons, including 5 interventions provided data for the analyses of this outcome. 548 patients $(39.68 \%)$ were assigned to lamivudine prophylaxis, $58(4.20 \%)$ to entecavir prophylaxis, 35 $(2.53 \%)$ to adefovir prophylaxis, $48(3.48 \%)$ to telbivudine prophylaxis and $692(50.11 \%)$ patients did not receive any prophylactic intervention.

Figure 3E illustrated the pooled estimates for the outcome of reducing the incidence of all causes of death. Lamivudine prophylaxis was statistically better than no prophylaxis (OR 0.35 , 95\% CI $0.20 \sim 0.59$ ). Other comparisons showed no statistical significance. However, there was a trend that all active interventions were better than control and telbivudine appeared to be more effective than lamivudine (OR 0.62, 95\% CI 0. $01 \sim 18.58$ ), adefovir

A

\begin{tabular}{|l|l|l|l|l|}
\hline Control & $0.10(0.06,0.14)$ & $0.07(0.02,0.20)$ & $0.04(0.01,0.17)$ & $0.02(0.01,0.05)$ \\
\hline $10.47(7.03,16.55)$ & Lamivudine & $0.77(0.26,2.04)$ & $0.43(0.12,1.74)$ & $0.23(0.10,0.49)$ \\
\hline $13.61(4.99,43.91)$ & $1.30(0.49,3.81)$ & Adefovir & $0.56(0.12,3.14)$ & $0.29(0.09,1.06)$ \\
\hline $24.50(6.01,94.04)$ & $2.34(0.58,8.44)$ & $1.79(0.32,8.69)$ & Telbivudine & $0.53(0.12,2.17)$ \\
\hline $45.75(20.40,111.09)$ & $4.38(2.06,9.53)$ & $3.40(0.94,11.04)$ & $1.89(0.46,8.50)$ & $0.05(0.00,0.71)$ \\
\hline $273.66(23.99,10135.32)$ & $26.29(2.35,954.82)$ & $20.13(1.41,830.28)$ & $11.44(0.74,542.60)$ & $5.94(0.49,231.17)$ \\
\hline
\end{tabular}

B

\begin{tabular}{|l|l|l|l|l|}
\hline Control & $0.26(0.02,9.38)$ & $0.12(0.07,0.17)$ & $0.05(0.01,0.22)$ & $0.02(0.01,0.07)$ \\
\hline $3.92(0.11,53.97)$ & Adefovir & $0.45(0.01,6.04)$ & $0.20(0.00,3.97)$ & $0.08(0.00,1.36)$ \\
\hline $8.59(5.84,13.35)$ & $2.21(0.17,82.04)$ & Lamivudine & $0.43(0.12,1.88)$ & $0.19(0.05,0.56)$ \\
\hline $19.88(4.48,77.95)$ & $5.09(0.25,222.66)$ & $2.33(0.53,8.19)$ & Telbivudine & $0.41(0.07,2.07)$ \\
\hline $46.71(14.33,185.10)$ & $13.21(0.74,595.04)$ & $5.36(1.78,19.97)$ & $2.43(0.48,14.64)$ & Entecavir \\
\hline
\end{tabular}

C

\begin{tabular}{|l|l|l|l|}
\hline Control & $0.35(0.21,0.60)$ & $0.28(0.01,2.36)$ & $0.11(0.01,0.58)$ \\
\hline $2.84(1.66,4.86)$ & Lamivudine & $0.79(0.03,6.58)$ & $0.32(0.04,1.65)$ \\
\hline $3.60(0.42,96.28)$ & $1.26(0.15,34.78)$ & Adefovir & $0.42(0.02,18.35)$ \\
\hline $8.88(1.73,90.38)$ & $3.11(0.61,27.37)$ & $2.40(0.05,44.79)$ & Entecavir \\
\hline
\end{tabular}

D

\begin{tabular}{|l|l|l|}
\hline Control & $0.23(0.15,0.33)$ & $0.08(0.03,0.25)$ \\
\hline $4.34(3.01,6.73)$ & Lamivudine & $0.36(0.12,1.08)$ \\
\hline $12.12(4.00,39.36)$ & $2.81(0.92,8.09)$ & Entecavir \\
\hline
\end{tabular}

E

\begin{tabular}{|l|l|l|l|l|}
\hline Control & $0.35(0.06,2.08)$ & $0.35(0.20,0.59)$ & $0.37(0.01,6.40)$ & $0.22(0.00,6.54)$ \\
\hline $2.83(0.48,18.01)$ & Adefovir & $1.01(0.18,5.71)$ & $1.06(0.02,29.11)$ & $0.62(0.01,26.69)$ \\
\hline $2.83(1.68,5.01)$ & $0.99(0.18,5.67)$ & Lamivudine & $1.05(0.03,17.67)$ & $0.62(0.01,18.58)$ \\
\hline $2.67(0.16,104.20)$ & $0.94(0.03,49.07)$ & $0.95(0.06,35.24)$ & Entecavir & $0.58(0.10,3.63)$ \\
\hline $4.63(0.15,257.91)$ & $1.62(0.04,116.81)$ & $1.62(0.05,85.58)$ & $1.72(0.28,10.02)$ & Telbivudine \\
\hline
\end{tabular}

Figure 3: Major clinical efficacy of all interventions according to network meta-analysis. Treatments are reported in efficacy order, which is from the least to the most efficacious treatment. The odds ratios (ORs) were estimated in upper and lower triangle comparing column-defining with row-defining treatment. ORs lower than 1 favors the column-defining treatment. Numbers in parentheses indicate 95\% confidence intervals. (A) HBV reactivation; (B) HBV-related hepatitis; (C) HBV-related death; (D) All causes of hepatitis; (E) All causes of death. 
(OR 0.62, 95\% CI $0.01 \sim 26.69$ ) as well as entecavir (OR 0.58, 95\% CI $0.10 \sim 3.63$ ). Figure 4A-4E showed the distribution of probabilities of each prophylactic interventions being ranked at each of the possible five positions. Telbivudine had the greatest probability (47\%) for being the best prophylactic option on reducing the incidence of overall death. The treatment of entecavir (34\%) shows the highest probability for being in the second ranking positions.

\section{Comparisons between traditional pairwise and Bayesian network meta-analyses}

Table 2 showed the results of traditional pairwise and Bayesian network meta-analyses. Although the pooled estimates for the outcome showed small differences, the 95\% CIs from traditional pairwise meta-analysis and network meta-analysis in general overlapped. Assessment of inconsistency by node splitting method between direct and indirect evidence was summarized in Table 3. The node-splitting method demonstrated inconsistency within the networks for the outcome of HBV-related hepatitis. No significant inconsistency within the networks for most treatment comparisons of the other three outcomes. As for the outcome of all causes of death, no closed loops were found. Therefore, inconsistency between direct and indirect evidence could not be assessed by the node splitting method, although the results of direct and indirect comparisons could be compatible for this outcome.

\section{DISCUSSION}

In this network meta-analysis, we evaluated the prophylactic efficacy of five oral NAs on chemotherapy- induced HBV reactivation and its related morbidity and mortality, including HBV-related hepatitis and death, all causes of hepatitis and mortality, in cancer patients with CHB infection. We found that tenofovir was the most effective agent to prevent $\mathrm{HBV}$ reactivation and prophylaxis with entecavir appeared to be the most potent intervention to reduce the incidence of $\mathrm{HBV}$ related morbidity and mortality.

It is well-known that liver injury resulting from $\mathrm{HBV}$ reactivation goes through two stages in $\mathrm{HBV}$ carrier receiving chemotherapy [55]. In the initial stage, chemotherapy-induced immune suppression may lead to remarkable HBV replication. Serum levels of HBV-DNA, HBV DNA polymerase, and hepatitis Be antigen ( $\mathrm{HBeAg}$ ) significantly increase and as a result, infected hepatocytes may suffer direct damage. The second stage comprises reconstitution of immune function after withdrawal of chemotherapy. The exaggerated immune response may mediate severe injury of infected hepatocytes, which clinically manifests as various HBV reactivation related complications, including hepatitis, liver failure and even death.

Since liver damage is closely relevant to $\mathrm{HBV}$ reactivation, prophylactic antiviral treatment during chemotherapy has been applied in the last decade. Lamivudine was initially available to prevent $\mathrm{HBV}$ reactivation clinically and several meta-analyses assessed the overall benefits of preventive lamivudine therapy in this population. Loomba et al. [56] showed that compared with no prophylaxis, lamivudine could significantly reduce the risk of $\mathrm{HBV}$ reactivation, $\mathrm{HBV}$-associated hepatitis by $79 \%$ in HBsAg positive patients who underwent chemotherapy. Similarly, a traditional meta-analysis [57]
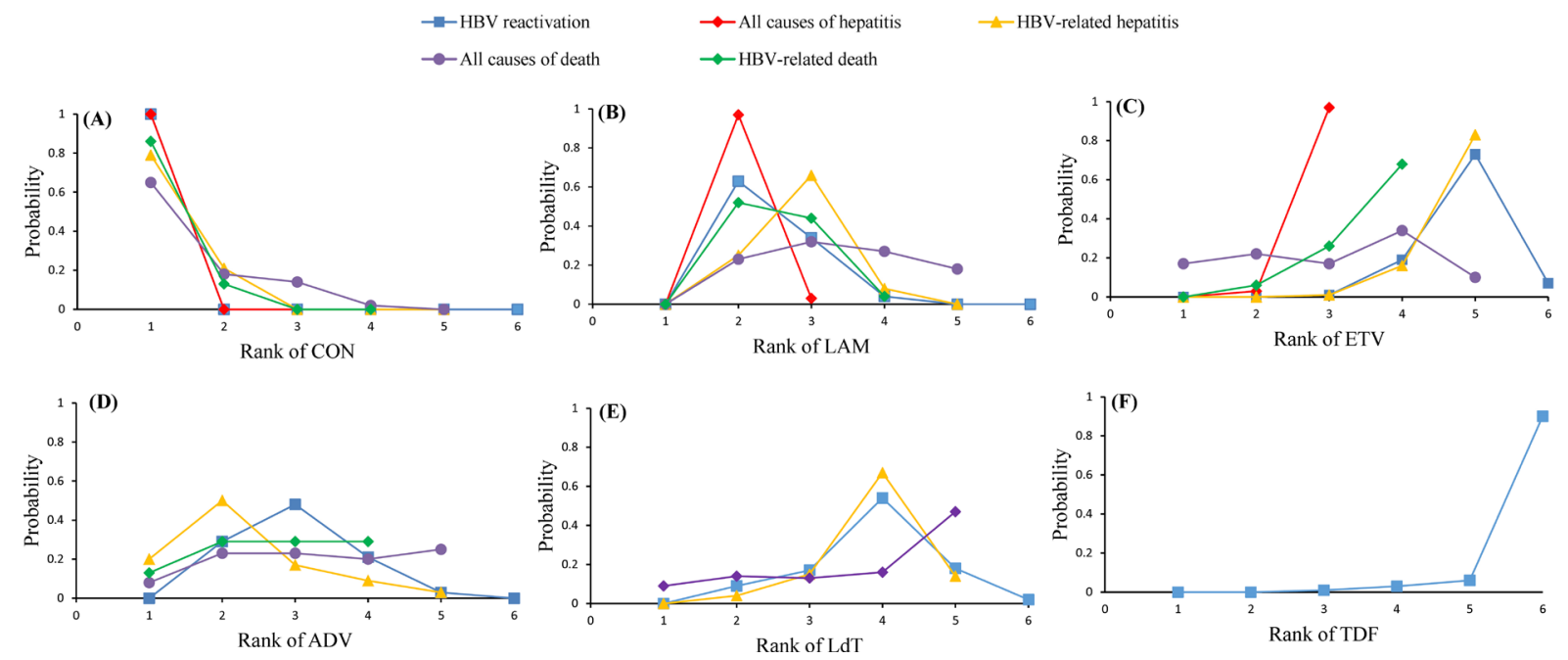

Figure 4: Rankograms showing probability of each strategy having each specific rank (1-6) for HBV reactivation, HBV-related hepatitis, HBV-related death, all causes of hepatitis and all causes of death. Ranking indicates the probability to be the best treatment, the second best, the third best and so on. Rank 1 is worst and rank N is best. (A) Rank of CON; (B) Rank of LAM; (C) Rank of ETV; (D) Rank of ADV; (E) Rank of LdT; (F) Rank of TDF. CON = control (no prophylaxis); LAM = lamivudine; ETV = entecavir; $\mathrm{ADV}=$ adefovir; $\mathrm{LdT}=$ telbivudine; $\mathrm{TDF}=$ tenofovir. 
Table 3: Assessment of inconsistency between direct and indirect evidence

Treatment comparisons

$P$ value of node-splitting method

\begin{tabular}{|c|c|}
\hline \multicolumn{2}{|l|}{ HBV reactivation } \\
\hline ADV vs. CON & 0.27 \\
\hline ADV vs. ETV & 0.61 \\
\hline LAM vs. CON & 0.15 \\
\hline ETV vs. CON & 0.4 \\
\hline ETV vs. TDF & 0.07 \\
\hline ADV vs. TDF & 0.11 \\
\hline ADV vs. LAM & 0.77 \\
\hline ETV vs. LAM & 0.22 \\
\hline \multicolumn{2}{|c|}{ HBV-related hepatitis } \\
\hline ETV vs. LAM & 0.01 \\
\hline ETV vs. CON & 0.02 \\
\hline LAM vs. CON & 0.02 \\
\hline \multicolumn{2}{|l|}{ HBV-related death } \\
\hline ADV vs. ETV & 0.02 \\
\hline ADV vs. LAM & 0.05 \\
\hline ADV vs. CON & 0.08 \\
\hline ETV vs. LAM & 0.90 \\
\hline ETV vs. CON & 0.77 \\
\hline LAM vs. CON & 0.77 \\
\hline \multicolumn{2}{|l|}{ All causes of hepatitis } \\
\hline ETV vs. LAM & 0.08 \\
\hline ETV vs. CON & 0.06 \\
\hline LAM vs. CON & 0.09 \\
\hline
\end{tabular}

$\mathrm{CON}=$ control (no prophylaxis); LAM = lamivudine; ETV = entecavir; ADV = adefovir; $\mathrm{LdT}=$ telbivudine; $\mathrm{TDF}=$ tenofovir.

including four RCTs also showed that lamivudine conferred a significantly more efficacious antiviral prophylaxis when compared with no prophylactic agent. With regards to other oral NAs, large scale RCTs with multiple comparator or meta-analysis have not been yet conducted to compare the effectiveness of different prophylactic interventions. For one RCT [10], prophylaxis with entecavir had a beneficial effect on lowering incidence with HBV-related hepatitis and HBV reactivation when compared with lamivudine among diffuse large B-cell lymphoma patients with CHB infection during chemotherapy. Another RCT [14] demonstrated that compared with lamivudine, the addition of adefovir resulted in similar efficacy in preventing chemotherapy-related HBV reactivation in $\mathrm{CHB}$ infected patients. The findings of these previous pairwise meta-analysis or RCTs are consistent with our findings.

Our meta-analysis has several strengths. Firstly, this is the largest meta-analysis with respect to this issue to date and we are confident that all correlative studies, including RCTs and cohort studies, have been properly assessed after extensive literature search and review. Furthermore, to our knowledge, this is also the first study which systematically assessed the prophylactic effect of all available oral NAs among CHB infection patients during chemotherapy. A traditional meta-analysis allows for only a direct comparison of individual pairs of intervention. However, this network meta-analysis combined total 3892 patients who received different prophylactic interventions and explored the effect of both direct and indirect comparisons between multiple interventions in a single analysis. In addition, after performing a Bayesian meta-analysis, we provided a rank order for prophylaxis strategies based on their capacity to reduce the incidence of HBV reactivation and HBV related events, which may provide an up to date scientific evidence to support clinicians in selecting oral NAs. Lastly, we conducted an inconsistency diagnostic analysis by node-splitting method for all loops to decrease concerns regarding potential inconsistency.

However, the results from our network meta-analysis need to be interpreted with caution for several reasons. First, there was some risk of bias in the included studies in terms of study design. Due to limited published RCTs, we also 
included the prospective and retrospective cohort studies for our study. But the methodological quality of these cohort studies was moderate to high by quality assessment. Besides that, heterogeneity of patients' characteristics, such as patient populations, definition of clinical outcome, duration of prophylaxis and follow-up, time of HBV reactivation and chemotherapy regimens used, also might result in bias in the analysis. However, the results of heterogeneity tests suggested low heterogeneity, which we believe are acceptable. Secondly, because of the low number of headto-head clinical trials comparing other anti-HBV agents except for lamivudine, the validity of our conclusions may be incomplete. Although tenofovir was suggested as the optimum treatment for primary outcome in this study, we were not able to assess the efficiency of tenofovir for secondary outcomes due to a lack of relevant data from the included trials. So we would recommend caution when interpreting the present conclusions. Thirdly, sample sizes assigned to each pair-wise comparison were small in many included studies. Fourthly, due to be unavailability of comprehensive data in most studies, we could not reliably evaluate the adverse effect of the different drugs.

In summary, this network meta-analysis suggests that chemotherapy with anti-HBV prophylaxis confers significant benefit over observation for patients with $\mathrm{CHB}$ infection. Among available NAs, tenofovir and entecavir may be the most effective treatment for prevention of chemotherapy-induced HBV reactivation and HBV-related morbidity and mortality.

\section{MATERIALS AND METHODS}

\section{Literature search}

We conducted a computerized literature search of PubMed, Embase, the Cochrane Library (prior to Mar 31, 2015) with the following keywords and subject terms: ["hepatitis B" (MeSH) or hepatitis B or $\mathrm{HBV}$ ] and [reactivation] and ["lamivudine" (MeSH) or "entecavir"(MeSH) or "adefovir" (MeSH) or "telbivudine" (MeSH) or "tenofovir" (MeSH)]. The literature search strategy was in accordance with PRISMA (Preferred Reporting Items for Systematic Reviews and MetaAnalyses) guideline [58]. References of retrieved articles and meeting abstracts were also screened. Case reports, editorials, letters and review articles were excluded. In case that a publication overlapped with other publication of the same trial, only the article with more details or the most recent article was adopted.

\section{Selection criteria}

Studies included in our meta-analysis satisfied all the following criteria: (1) studies were RCTs or retrospective or prospective cohort studies with controls (concurrent or historical); (2) study population was hematological or solid cancers patients with HBsAg positive undergoing chemotherapy or HSCT; (3) studies assessed the effectiveness of prophylactic therapy with one or more of the five oral NAs, including lamivudine, entecavir, adefovir, telbivudine and tenofovir, on prevention of HBV reactivation or HBV-related morbidity and mortality during chemotherapy. Exclusion criteria were: (1) non-cancer patients receiving immunosuppression therapy, including organ transplantation, inflammatory bowel disease and autoimmune diseases patients; (2) patients co-infecting human immunodeficiency virus (HIV) or other hepatitis viruses (hepatitis $\mathrm{C}$ virus [HCV], hepatitis D virus [HDV]); (3) patients with past HBV infection [HBsAg negative and hepatitis B core antibody ( $\mathrm{HBcAb}$ ) positive]; (4) patients receiving oral NAs treatment before. When a paper did not provide relevant data, or the case that the provided data were not sufficient, we contacted the corresponding authors by e-mail to obtain the raw data.

The primary outcome of this study was incidence of $\mathrm{HBV}$ reactivation, which was defined as an increase in HBV-DNA level to 10-fold or more when compared with baseline level, or appearance of HBV-DNA in previously negative patient or an absolute increase of HBV-DNA that exceeded $10^{9} \mathrm{ge} / \mathrm{mL}$ in the absence of other systemic infection[59]. The secondary outcome measures included: (1) all causes of hepatitis, defined as more than 3-fold increase in alanine aminotransferase (ALT) that exceeded the upper normal limit (UNL) or an absolute increase of ALT to over $100 \mathrm{IU} / \mathrm{L}$; (2) HBV-related hepatitis, defined as more than 3-fold increase in ALT that exceeded UNL and a 10 -fold increase in serum HBV-DNA level in the absence of other apparent causes of hepatitis; (3) all causes of death; (4) HBV-related death, defined as death of a patient who had documented HBV reactivation without other apparent causes of death.

\section{Data extraction and study quality}

Two reviewers (Min-Yue Zhang, Gui-Qi Zhu) independently assessed the full manuscripts or abstracts of eligible studies and extracted data and outcomes using an electronic standard form. The following information from each study was summarized: (1) first author, (2) year of publication, (3) country, (4) study design, (5) types of cancers, (6) prophylactic interventions and (7) patients' characteristics, including ages, total numbers of patients, numbers of male patients, numbers of events, duration of prophylaxis and follow up. Any conflicts regarding data extraction were resolved by an additional investigator, Ming-Hua Zheng.

\section{Quality assessment}

Two reviewers (Min-Yue Zhang, Gui-Qi Zhu) independently assessed the quality of each included studies. Newcastle-Ottawa Quality Assessment Scale was adopted to assess the methodological quality of prospective or retrospective cohort studies. Three major components of 
each study, including patient selection, comparability of interventions and observation group, and assessment of outcome, were examined (Supplementary Table 2). Cochrane Risk of Bias Tool was adopted to assess the methodological quality of included RCTs [60]. This tool included the following items: sequence generation for the randomization of subjects, allocation of concealment of treatment, blinding, incomplete outcome data, selective outcome reporting and other sources of bias. Trials with high or unclear risk for bias for any one of the first three components were considered as trials with high risk of bias. Otherwise, they were regarded as trials with low risk of bias.

\section{Data analysis}

Firstly the pairwise meta-analysis was conducted using Stata software (version 10.0, StataCorp, College Station, TX). Then a network meta-analysis within a Bayesian framework was conducted using Markov chain Monte Carlo methods in WinBUGS (Medical Research Council Biostatistics Unit, Cambridge, United Kingdom). The methods of pairwise meta-analysis and network metaanalysis were detailed in our previous publications [61-65].

\section{Abbreviations}

NA: nucleos(t)ide analogue; HBV: hepatitis B virus; $\mathrm{CHB}$ infection: chronic $\mathrm{HBV}$ infection; OR: odds ratio; $\mathrm{CI}$ : confidence interval; $\mathrm{HBsAg}$ : hepatitis $\mathrm{B}$ surface antigen; HBeAg: hepatitis B e antigen; ALT: alanine aminotransferase; Allo-HSCT: allogeneic hematopoietic stem cell transplantation; RCT: randomized controlled trial; HIV: human immunodeficiency virus; HCV: hepatitis $\mathrm{C}$ virus; $\mathrm{HDV}$ : hepatitis D virus; $\mathrm{HBcAb}$ : hepatitis $\mathrm{B}$ core antibody; UNL: upper normal limit; CON: control (no prophylaxis); LAM: lamivudine; ETV: entecavir; ADV: adefovir; LdT: telbivudine; TDF: tenofovir.

\section{ACKNOWLEDGMENTS}

Author contributions: Zhang MY, Zhu GQ, Shi KQ, Chen FY and Zheng MH designed the study. Zhang MY and Zhu GQ screened studies and extracted data. Zhu GQ and Zheng $\mathrm{JN}$ did the statistical analyses. Cheng $\mathrm{Z}$ and Zou ZL prepared figures. Zhang MY, Zhu GQ, Huang HH, Chen FY and Zheng MH reviewed the results, interpreted data, and wrote the manuscript. All authors saw and approved the final version of the paper.

\section{FUNDING}

This work was supported by grants from the National Natural Science Foundation of China (81500665), Zhejiang Engineering Research Center of Intelligent Medicine (2016E10011) and Project of New Century 551 Talent Nurturing in Wenzhou and Project of Further Accelerating
Development of Traditional Chinese Medicine in Shanghai (ZY3-CCCX-3-3037).

\section{CONFLICTS OF INTEREST}

The authors report no declarations of interest.

\section{REFERENCES}

1. Ott JJ, Stevens GA, Groeger J, Wiersma ST. Global epidemiology of hepatitis B virus infection: new estimates of age-specific HBsAg seroprevalence and endemicity. Vaccine. 2012; 30:2212-2219.

2. Lin GN, Peng JW, Xiao JJ, Liu DY, Xia ZJ. Hepatitis B virus reactivation in hepatitis $\mathrm{B}$ surface antigen seropositive patients with metastatic non-small cell lung cancer receiving cytotoxic chemotherapy: the efficacy of preemptive lamivudine and identification of risk factors. Med Oncol. 2014; $31: 119$.

3. Kim SJ, Hsu C, Song YQ, Tay K, Hong XN, Cao J, Kim JS, Eom HS, Lee JH, Zhu J, Chang KM, Reksodiputro AH, Tan D, et al. Hepatitis B virus reactivation in B-cell lymphoma patients treated with rituximab: analysis from the Asia Lymphoma Study Group. Eur J Cancer. 2013; 49:3486-3496.

4. Lee GW, Ryu MH, Lee JL, Oh S, Kim E, Lee JH, Kim SB, Kim SW, Suh C, Lee KH, Kim WK, Lee JS, Kang YK. The prophylactic use of lamivudine can maintain doseintensity of adriamycin in hepatitis-B surface antigen (HBs Ag)-positive patients with Non-Hodgkin's lymphoma who receive cytotoxic chemotherapy. J Korean Med Sci. 2003; 18:849-854.

5. Nagamatsu $\mathrm{H}$, Itano $\mathrm{S}$, Nagaoka $\mathrm{S}$, Akiyoshi J, Matsugaki $\mathrm{S}$, Kurogi J, Tajiri N, Yamasaki S, Koga H, Torimura T, Kumashiro R, Sata M. Prophylactic lamivudine administration prevents exacerbation of liver damage in $\mathrm{HBe}$ antigen positive patients with hepatocellular carcinoma undergoing transhepatic arterial infusion chemotherapy. Am J Gastroenterol. 2004; 99:2369-2375.

6. Lau GK, Yiu HH, Fong DY, Cheng HC, Au WY, Lai LS, Cheung M, Zhang HY, Lie A, Ngan R, Liang R. Early is superior to deferred preemptive lamivudine therapy for hepatitis $\mathrm{B}$ patients undergoing chemotherapy. Gastroenterology. 2003; 125:1742-1749.

7. Lee HJ, Kim DY, Keam B, Lee JH, Han SW, Oh DY, Yoon JH, Kim TY, Kim YJ, Lee KW, Kim JW, Jeong SH, Lee JS, et al. Lamivudine prophylaxis for hepatitis B virus carrier patients with breast cancer during adjuvant chemotherapy. Breast cancer. 2014; 21:387-393.

8. Jang JW, Choi JY, Bae SH, Yoon SK, Chang UI, Kim CW, Cho SH, Han JY, Lee YS. A randomized controlled study of preemptive lamivudine in patients receiving transarterial chemo-lipiodolization. Hepatology. 2006; 43:233-240.

9. Liaw YF, Kao JH, Piratvisuth T, Chan HLY, Chien RN, Liu CJ, Gane E, Locarnini S, Lim SG, Han KH, Amarapurkar D, 
Cooksley G, Jafri W, et al. Asian-Pacific consensus statement on the management of chronic hepatitis B: A 2012 update. Hepatol Int. 2012; 6:531-561.

10. Huang H, Li X, Zhu J, Ye S, Zhang H, Wang W, Wu X, Peng J, Xu B, Lin Y, Cao Y, Li H, Lin S, et al. Entecavir vs lamivudine for prevention of hepatitis $\mathrm{B}$ virus reactivation among patients with untreated diffuse large B-cell lymphoma receiving R-CHOP chemotherapy: a randomized clinical trial. JAMA. 2014; 312:2521-2530.

11. Chen MY, Liu CJ, Kao JH. Prophylaxis nucleot(s) ides in chronic hepatitis B infected patients undergoing chemotherapy for malignancies. Hepatol Int. 2013; 7: S226-S227.

12. Hsu C, Hsiung CA, Su IJ, Hwang WS, Wang MC, Lin SF, Lin TH, Hsiao HH, Young JH, Chang MC, Liao YM, Li CC, $\mathrm{Wu} \mathrm{HB}$, et al. A revisit of prophylactic lamivudine for chemotherapy-associated hepatitis B reactivation in nonHodgkin's lymphoma: a randomized trial. Hepatology. 2008; 47:844-853.

13. Long M, Jia W, Li S, Jin L, Wu J, Rao N, Feng H, Chen K, Deng H, Liu F, Su F, Song E. A single-center, prospective and randomized controlled study: Can the prophylactic use of lamivudine prevent hepatitis B virus reactivation in hepatitis B s-antigen seropositive breast cancer patients during chemotherapy? Breast Cancer Res Treat. 2011; 127:705-712.

14. Ho EY, Yau T, Rousseau F, Heathcote EJ, Lau GK. Preemptive adefovir versus lamivudine for prevention of hepatitis B reactivation in chronic hepatitis B patients undergoing chemotherapy. Hepatol Int. 2015; 9:224-230.

15. Lim LL, Wai CT, Lee YM, Kong HL, Lim R, Koay E, Lim SG. Prophylactic lamivudine prevents hepatitis B reactivation in chemotherapy patients. Aliment Pharmacol Ther. 2002; 16:1939-1944.

16. Persico M, De Marino F, Russo GD, Morante A, Rotoli B, Torella R, De Renzo A. Efficacy of lamivudine to prevent hepatitis reactivation in hepatitis $\mathrm{B}$ virus-infected patients treated for non-Hodgkin lymphoma. Blood. 2002; 99:724-725.

17. Leaw SJ, Yen CJ, Huang WT, Chen TY, Su WC, Tsao CJ. Preemptive use of interferon or lamivudine for hepatitis B reactivation in patients with aggressive lymphoma receiving chemotherapy. Ann Hematol. 2004; 83:270-275.

18. Jia J, Lin F. [Lamivudine therapy for prevention of chemotherapy-induced reactivation of hepatitis B virus]. Chin J Hepatol. 2004; 12:628-629.

19. Ozguroglu M, Bilici A, Turna H, Serdengecti S. Reactivation of hepatitis B virus infection with cytotoxic therapy in non-Hodgkin's lymphoma. Med Oncol. 2004; 21:67-72.

20. Lim ST, Fei G, Quek R, Lim LC, Lee LH, Yap SP, Loong S, Tao M. The relationship of hepatitis B virus infection and non-Hodgkin's lymphoma and its impact on clinical characteristics and prognosis. Eur J Haematol. 2007; 79:132-137.
21. Chen Y, Xu LP, Liu KY, Liu DH, Han W, Chen H, Zhang YC, Chen YH, Huang XJ. [A clinical study of allo-hematopoietic stem cell transplantation in HBsAg positive leukemia]. Chin J Intern Med. 2008; 47:316-319.

22. Tsutsumi Y, Yamamoto Y, Tanaka J, Asaka M, Imamura M, Masauzi N. Prevention of hepatitis B virus reactivation under rituximab therapy. Immunotherapy. 2009; 1:1053-1061.

23. Eren OO, Artac M, Boruban MC, Yavas O, Arslan U, Basaranoglu M. Chemotherapy-induced Hepatitis B virus reactivation in $\mathrm{HbsAg}$ positive cancer patients: a single center experience. Med Oncol. 2009; 26:386-392.

24. Yeo W, Chan TC, Leung NW, Lam WY, Mo FK, Chu MT, Chan HL, Hui EP, Lei KI, Mok TS, Chan PK. Hepatitis B virus reactivation in lymphoma patients with prior resolved hepatitis B undergoing anticancer therapy with or without rituximab. J Clin Oncol. 2009; 27:605-611.

25. Koo YX, Tan DS, Tan IB, Tao M, Chow WC, Lim ST. Hepatitis $\mathrm{B}$ virus reactivation and role of antiviral prophylaxis in lymphoma patients with past hepatitis B virus infection who are receiving chemoimmunotherapy. Cancer. 2010; 116:115-121.

26. Topcuoglu P, Soydan E, Idilman R, Arslan O, Ozcan M, Ilhan O, Beksac M, Arat M, Gurman G. Allogeneic hematopoetic cell transplantation in patients positive for hepatitis B surface antigen. Biol Blood Marrow Transplant. 2010; 16:S288.

27. Pei SN, Chen $\mathrm{CH}$, Lee CM, Wang MC, Ma MC, Hu TH, Kuo CY. Reactivation of hepatitis B virus following rituximab-based regimens: a serious complication in both HBsAg-positive and HBsAg-negative patients. Ann Hematol. 2010; 89:255-262.

28. Sohn BS, Ahn JH, Jung KH, Ahn SH, Son BH, Gong G, Kim SB. Updated longitudinal data on acute exacerbation of chronic hepatitis B in patients with breast cancer receiving anthracycline-based adjuvant chemotherapy: therapeutic vs. pre-emptive use of lamivudine. Jpn J Clin Oncol. 2011; 41:1059-1066.

29. Yun J, Kim KH, Kang ES, Gwak GY, Choi MS, Lee JE, Nam SJ, Yang JH, Park YH, Ahn JS, Im YH. Prophylactic use of lamivudine for hepatitis B exacerbation in post-operative breast cancer patients receiving anthracycline-based adjuvant chemotherapy. Br J Cancer. 2011; 104:559-563.

30. Yan Y, Zhang JW, Zhao HY, Huang Y, Hu ZH, Zhang L. [Retrospective analysis of the correlation between"hepatitis $B$ virus infection and chemotherapy-induced hepatic injury in patients with lung cancer]. Chin J Clin Oncol. 2012; 39:465-469.

31. Mya DH, Han ST, Linn YC, Hwang WY, Goh YT, Tan DC. Risk of hepatitis B reactivation and the role of novel agents and stem-cell transplantation in multiple myeloma patients with hepatitis B virus (HBV) infection. Ann Oncol. 2012; 23:421-426.

32. Chen XQ, Peng JW, Lin GN, Li M, Xia ZJ. The effect of prophylactic lamivudine on hepatitis $\mathrm{B}$ virus reactivation in HBsAg-positive patients with diffuse large B-cell lymphoma 
undergoing prolonged rituximab therapy. Med Oncol. 2012; 29:1237-1241.

33. Wang YH, Fan L, Wang L, Zhang R, Xu J, Fang C, Li JY, $\mathrm{Xu}$ W. Efficacy of prophylactic lamivudine to prevent hepatitis B virus reactivation in B-cell lymphoma treated with rituximab-containing chemotherapy. Support Care Cancer. 2013; 21:1265-1271.

34. Nishida T, Hiramatsu N, Mizuki M, Nagatomo I, Kida H, Tazumi K, Shinzaki S, Miyazaki M, Yakushijin T, Tatsumi T, Iijima H, Kiso S, Kanto T, et al. Managing hepatitis B virus carriers with systemic chemotherapy or biologic therapy in the outpatient clinic. Hepatol Res. 2013; 43:339-346.

35. Chen FW, Coyle L, Jones BE, Pattullo V. Entecavir versus lamivudine for hepatitis B prophylaxis in patients with haematological disease. Liver Int 2013; 33:1203-1210.

36. Li HR, Huang JJ, Guo HQ, Zhang X, Xie Y, Zhu HL, Zhai LZ, Pu XX, Huang Y, Guo CC, Lin TY. Comparison of entecavir and lamivudine in preventing hepatitis $\mathrm{B}$ reactivation in lymphoma patients during chemotherapy. $\mathrm{J}$ Viral Hepat. 2011; 18:877-883.

37. Min YW, Kim NJ, Gwak GY, Paik YH, Choi MS, Koh KC, Paik SW, Yoo BC, Lee JH. Lamivudine versus entecavir for prevention of hepatitis $\mathrm{B}$ virus reactivation in hepatitis $\mathrm{B}$ surface antigen positive non-hepatic cancer patients receiving chemotherapy. Hepatology. 2012; 56:387A-388A.

38. Ling WH, Soe PP, Pang AS, Lee SC. Hepatitis B virus reactivation risk varies with different chemotherapy regimens commonly used in solid tumours. Br J Cancer. 2013; 108:1931-1935.

39. Lau GK, He ML, Fong DY, Bartholomeusz A, Au WY, Lie AK, Locarnini S, Liang R. Preemptive use of lamivudine reduces hepatitis B exacerbation after allogeneic hematopoietic cell transplantation. Hepatology. 2002; 36:702-709.

40. Li YH, He YF, Jiang WQ, Wang FH, Lin XB, Zhang L, Xia ZJ, Sun XF, Huang HQ, Lin TY, He YJ, Guan ZZ. Lamivudine prophylaxis reduces the incidence and severity of hepatitis in hepatitis B virus carriers who receive chemotherapy for lymphoma. Cancer. 2006;106:1320-1325.

41. Hsiao LT, Chiou TJ, Liu JH, Chu CJ, Lin YC, Chao TC, Wang WS, Yen CC, Yang MH, Tzeng CH, Chen PM. Extended lamivudine therapy against hepatitis B virus infection in hematopoietic stem cell transplant recipients. Biol Blood Marrow Transplant. 2006; 12:84-94.

42. Cil T, Altintas A, Pasa S, Bayan K, Ozekinci T, Isikdogan A. Lamivudine for the prevention of hepatitis $\mathrm{B}$ virus reactivation in hepatitis-B surface antigen (HBSAG) seropositive cancer patients undergoing cytotoxic chemotherapy. Leuk Lymphoma. 2008; 49:939-947.

43. Huang H, Cai Q, Lin T, Lin X, Liu Y, Gao Y, Peng R. Lamivudine for the prevention of hepatitis $\mathrm{B}$ virus reactivation after high-dose chemotherapy and autologous hematopoietic stem cell transplantation for patients with advanced or relapsed non-Hodgkin's lymphoma single institution experience. Expert Opin Pharmacother. 2009; 10:2399-2406.
44. Shibolet O, Ilan Y, Gillis S, Hubert A, Shouval D, Safadi R. Lamivudine therapy for prevention of immunosuppressiveinduced hepatitis B virus reactivation in hepatitis B surface antigen carriers. Blood. 2002; 100:391-396.

45. Idilman R, Arat M, Soydan E, Toruner M, Soykan I, Akbulut H, Arslan O, Ozcan M, Turkyilmaz AR, Bozdayi M, Karayalcin S, Van Thiel DH, Ozden A, et al. Lamivudine prophylaxis for prevention of chemotherapy-induced hepatitis B virus reactivation in hepatitis B virus carriers with malignancies. J Viral Hepat. 2004;11:141-147.

46. Tsai SH, Dai MS, Yu JC, Ho CL, Chen YC, Wu YY, Chang PY, Kao WY, Chao TY. Preventing chemotherapyinduced hepatitis B reactivation in breast cancer patients: a prospective comparison of prophylactic versus deferred preemptive lamivudine. Supportive care cancer. 2011; 19:1779-1787.

47. Gentile G, Russo E, De Angelis F, Di Rocco A, Fama A, Micozzi A, Pulsoni A, Martelli M, Mauro FR, Iori AP, Petrucci T, Vitale A, Antonelli G, et al. Efficacy and safety of long term tenofovir in high risk patients with hematological malignancies (HM) to prevent Hepatitis B Virus (HBV) reactivation after immunosuppressive therapies in real life. Hepatology. 2014; 60:1008A.

48. Yeo W, Chan PK, Ho WM, Zee B, Lam KC, Lei KI, Chan AT, Mok TS, Lee JJ, Leung TW, Zhong S, Johnson PJ. Lamivudine for the prevention of hepatitis $\mathrm{B}$ virus reactivation in hepatitis B s-antigen seropositive cancer patients undergoing cytotoxic chemotherapy. J Clin Oncol. 2004; 22:927-934.

49. Yeo W, Ho WM, Hui P, Chan PK, Lam KC, Lee JJ, Johnson PJ. Use of lamivudine to prevent hepatitis B virus reactivation during chemotherapy in breast cancer patients. Breast Cancer Res Treat. 2004; 88:209-215.

50. Dai MS, Chao TY, Kao WY, Shyu RY, Liu TM. Delayed hepatitis B virus reactivation after cessation of preemptive lamivudine in lymphoma patients treated with rituximab plus CHOP. Ann Hematol. 2004; 83:769-774.

51. Yeo W, Hui EP, Chan AT, Ho WM, Lam KC, Chan PK, Mok TS, Lee JJ, Mo FK, Johnson PJ. Prevention of hepatitis B virus reactivation in patients with nasopharyngeal carcinoma with lamivudine. Am J Clin Oncol. 2005; 28:379-384.

52. Hui CK, Cheung WW, Au WY, Lie AK, Zhang HY, Yueng YH, Wong BC, Leung N, Kwong YL, Liang R, Lau GK. Hepatitis $\mathrm{B}$ reactivation after withdrawal of pre-emptive lamivudine in patients with haematological malignancy on completion of cytotoxic chemotherapy. Gut. 2005; 54:1597-1603.

53. Choi J, An J, Shim JH, Kim H-D, Ha Y-J, Jun M-J, Yang YJ, Lee SB, Ae Kim G, Yang JE, Park EJ, Lee D, Kim KM, et al. Risk and Prediction of Hepatitis B Reactivation in Inactive Carriers Receiving Pre-emptive Antiviral Therapy During Cancer Chemotherapy: 1853. [Abstract]. Hepatology October. 2014; 60:1088A-1089A.

54. Yoo JJ, Kim HY, Lee JH, Yu SJ, Kim YJ, Yoon JH, Lee HS. Comparison of lamivudine, telbivudine, and entecavir as antiviral prophylaxis for patients with Hepatitis B undergoing cytotoxic chemotherapy. Hepatology. 2012; 56:631A. 
55. Xunrong L, Yan AW, Liang R, Lau GK. Hepatitis B virus (HBV) reactivation after cytotoxic or immunosuppressive therapy - pathogenesis and management. Rev Med Virol. 2001; 11:287-299.

56. Loomba R, Rowley A, Wesley R, Liang TJ, Hoofnagle JH, Pucino F, Csako G. Systematic review: the effect of preventive lamivudine on hepatitis B reactivation during chemotherapy. Ann Intern Med. 2008; 148:519-528.

57. Perrillo RP, Gish R, Falck-Ytter YT. American Gastroenterological Association Institute technical review on prevention and treatment of hepatitis B virus reactivation during immunosuppressive drug therapy. Gastroenterology. 2015; 148:221-244.

58. Hutton B, Salanti G, Caldwell DM, Chaimani A, Schmid CH, Cameron C, Ioannidis JP, Straus S, Thorlund K, Jansen JP, Mulrow C, Catala-Lopez F, Gotzsche PC, et al. The PRISMA extension statement for reporting of systematic reviews incorporating network meta-analyses of health care interventions: checklist and explanations. Ann Intern Med. 2015; 162:777-784.

59. Kohrt HE, Ouyang DL, Keeffe EB. Systematic review: lamivudine prophylaxis for chemotherapy-induced reactivation of chronic hepatitis $\mathrm{B}$ virus infection. Aliment Pharmacol Ther. 2006; 24:1003-1016.

60. Higgins JP, Altman DG, Gotzsche PC, Juni P, Moher D, Oxman AD, Savovic J, Schulz KF, Weeks L, Sterne JA. The Cochrane Collaboration's tool for assessing risk of bias in randomised trials. BMJ. 2011; 343:d5928.
61. Zhou YY, Zhu GQ, Wang Y, Zheng JN, Ruan LY, Cheng Z, Hu B, Fu SW, Zheng MH. Systematic review with network meta-analysis: statins and risk of hepatocellular carcinoma. Oncotarget. 2016. doi: 10.18632/oncotarget.7832.

62. Zhu GQ, Shi KQ, Huang GQ, Wang LR, Lin YQ, Braddock M, Chen YP, Zhou MT, Zheng MH. A network meta-analysis of the efficacy and side effects of udca-based therapies for primary sclerosing cholangitis. Oncotarget. 2015; 6: 26757-26769. doi: 10.18632/oncotarget.5610.

63. Zhu GQ, Shi KQ, Huang S, Wang LR, Lin YQ, Huang GQ, Chen YP, Braddock M, Zheng MH. Systematic review with network meta-analysis: the comparative effectiveness and safety of interventions in patients with overt hepatic encephalopathy. Aliment Pharmacol Ther. 2015; 41: 624-635.

64. Zhu GQ, Shi KQ, Yu HJ, He SY, Braddock M, Zhou MT, Chen YP, Zheng MH. Optimal adjuvant therapy for resected hepatocellular carcinoma: a systematic review with network meta-analysis. Oncotarget. 2015; 6:18151-18161. doi: 10.18632/oncotarget.4098.

65. Zhu GQ, Zou ZL, Zheng JN, Chen DZ, Zou TT, Shi KQ, Zheng MH. Systematic Review and Network MetaAnalysis of Randomized Controlled Trials: Comparative Effectiveness and Safety of Direct-Acting Antiviral Agents for Treatment-Naive Hepatitis C Genotype 1. Medicine. 2016; 95:e3004. 